\title{
Neutron Detection in a High-Gamma Field Using Solution-Grown Stilbene
}

\author{
M. M. Bourne ${ }^{1}$, S. D. Clarke ${ }^{1}$, N. Adamowicz ${ }^{1}$, S. A. Pozzi ${ }^{1}$, N. Zaitseva ${ }^{2}$, L. Carman ${ }^{2}$ \\ ${ }^{1}$ University of Michigan, Ann Arbor, MI 48109, USA \\ ${ }^{2}$ Lawrence Livermore National Laboratory, Livermore, CA 94550, USA \\ Email: mmbourne@umich.edu,nicka@umich.edu, clarkesd@umich.edu, pozzisa@umich.edu, zaitseva1@1lnl.gov, \\ carman1@1lnl.gov
}

\begin{abstract}
A solution-based technique for growing large-volume stilbene scintillators was developed in 2013; crystals up to diameters of 10 centimeters, or larger, have been grown while preserving excellent pulse shape discrimination (PSD) properties. The goal of this study is to evaluate the PSD capabilities of 5.08 by $5.08-\mathrm{cm}$ stilbene crystals grown by Lawrence Livermore National Laboratory and Inrad Optics when exposed to a 1000-to-1 gamma ray-neutron ratio and operating at a $100-\mathrm{kHz}$ count rate. Results were compared to an equivalent EJ-309 liquid scintillation detector. ${ }^{252} \mathrm{Cf}$ neutron pulses were recorded in two experiments where ${ }^{60} \mathrm{Co}$ and ${ }^{137} \mathrm{Cs}$ sources created the high-gamma field. The high count rate created numerous double pulses that were cleaned using fractional and template approaches designed to remove double pulses while preserving neutron counts. PSD was performed at a threshold of $42 \mathrm{keVee}(440-\mathrm{keV}$ proton) for stilbene and $60 \mathrm{keVee}(610 \mathrm{-keV}$ proton) for EJ-309 liquid. The lower threshold in stilbene resulted in a neutron intrinsic efficiency of approximately $14.5 \%, 10 \%$ higher than EJ-309 liquid, for bare ${ }^{252} \mathrm{Cf}$ and $13 \%$ for ${ }^{252} \mathrm{Cf}$ in the high-gamma field. Despite the lower threshold, the gamma misclassification rate in stilbene was approximately $3 \times$ $10^{-6}$, nearly a factor-of-five lower than what we found with the EJ-309 liquid.
\end{abstract}

Keywords: stilbene scintillator, liquid scintillator, pulse shape discrimination, neutron detection

\section{Introduction}

The ${ }^{3} \mathrm{He}$ shortage has motivated efforts to finding more cost-effective neutron-detection alternatives for homeland security, well logging, fusion research, and nuclear nonproliferation applications [1]. Organic scintillators, including stilbene and liquids such as EJ-309, feature fastneutron counting from nanosecond-level pulse timing, good efficiency, and excellent pulse-shape discrimination (PSD) for identification of pulses originating from neutron and gamma ray interactions [2, 3, 4]. A solution-based stilbene synthesis technique was developed in 2013 by Lawrence Livermore National Laboratory (LLNL) that allows us to consistently grow crystals up to 10 centimeters in diameter. These crystals are significantly larger than those from traditional meltbased growth techniques, and improve the scintillator's light production from incident radiation [5]. This results in scintillators with PSD capabilities that compare favorably to liquid organic scintillators [5, 6]. Recently, this solution-based stilbene is commercially available from Inrad Optics (IO) [7]. Figure 1 shows photographs of a solution-based stilbene crystal and assembly developed at the University of Michigan.

The gamma ray and neutron detection capability of organic scintillators becomes a disadvantage for neutron counting when the gamma ray count rate becomes large. While ${ }^{3} \mathrm{He}$ is unaffected by gamma ray background, the near-simultaneous detection of two or more gamma rays in the same scintillator is likely to be misclassified as a neutron by charge integration PSD. These double pulses must be removed from data analysis for accurate neutron counting. Double pulse cleaning, however, is never perfect, and this results in the loss of "good" neutron pulses, thus lowering the detector's neutron detection efficiency. This work seeks to determine how well 
stilbene can measure unshielded ${ }^{252} \mathrm{Cf}$ neutrons in fields of 1,000 external gamma rays per ${ }^{252} \mathrm{Cf}$ neutron. Previous measurements have shown that, when operated at a 36-keVee threshold, the LLNL stilbene could function in a field of $1,000{ }^{60} \mathrm{Co}$ gammas per ${ }^{252} \mathrm{Cf}$ neutron and $60-\mathrm{kHz}$ count rate with intrinsic neutron efficiency greater than $20 \%$ and gamma misclassification rate of $3 \times 10^{-5}$ and using no lead shielding [8]. By implementing a hybrid approach to double-pulse cleaning, this work improves the misclassification rate reported in [8], while sacrificing a small amount of intrinsic neutron efficiency. Both ${ }^{60} \mathrm{Co}$ and ${ }^{137} \mathrm{Cs}$ were used in separate experiments to increase the gamma ray count rate to determine how the cleaning approach is affected by incident gamma energy. The measured count rate for these experiments was approximately $100 \mathrm{kHz}$. The LLNL and Inrad Optic stilbene assemblies were compared to an EJ-309 liquid scintillator assembly. Comparison of the crystals obtained from different sources was assumed to be helpful in evaluation of variations in performance of individual crystals produced using the same technique and initial raw material [9].

Fig. 1.

\section{Experimental Setup}

The LLNL and Inrad Optic stilbene scintillation crystals are right circular cylinders with a nominal diameter of $5.08 \mathrm{~cm}$ and a length of $5.08 \mathrm{~cm}$. These crystals were mounted using optical grease onto separate 5.08-cm diameter PMTs with model number ETL 9214A or 9214B [10]. The EJ-309 liquid scintillation cell, a right circular cylinder, had the same dimensions as the stilbene crystals [11]. This detector assembly was manufactured by SCIONIX using the same 5.08-cm diameter PMT model and equivalent base electronics as that used for the stilbene detector. Anode signals from each detector were digitized using the CAEN DT5720 digitizer, which has a 250-MHz sampling rate, 2 -Volt dynamic range, and 12-bit nominal vertical resolution. Pulse waveforms were sampled over a 400-ns data acquisition window: this window length is long enough to obtain good PSD.

\section{$2.1{ }^{252}$ Cf and GammaRay Measurements}

The stilbene and EJ-309 liquid assemblies were gain-matched to equal electron-equivalent light output. A one-microCurie ${ }^{137} \mathrm{Cs}$ source was used for calibrating each detector; the gain for each detector was set to align the ${ }^{137} \mathrm{Cs}$ Compton edge — chosen at $80 \%$ of the edge maximum - at 0.4 Volts. Figure 2 shows the ${ }^{137} \mathrm{Cs}$ pulse height spectra recorded in separate calibration measurements for each assembly.

Fig. 2.

Figure 3 shows the measurement geometry used for the stilbene and EJ-309 scintillators. Separate measurements were performed using either ${ }^{60} \mathrm{Co}$ or ${ }^{137} \mathrm{Cs}$ to create the high-gamma ray field. In each measurement, the gamma ray source was positioned such that the count rate for each scintillator was approximately $100 \mathrm{kHz}$; attempting to increase the count rate above this value would lead to data loss (a limitation of the digitization system). The ${ }^{252} \mathrm{Cf}$ source, with neutron emission rate of approximately 141,000 neutrons per second, was then positioned such that at least 1,000 gamma rays were incident on the scintillator face per incident ${ }^{252} \mathrm{Cf}$ neutron. Measurements 
were performed using the ${ }^{252} \mathrm{Cf}$ and gamma ray sources together and separately. Finally, background data were recorded overnight. Table I shows the source position for each measurement set recorded by each detector. The measurement threshold was set to $42 \mathrm{keVee}$ for each detector.

Fig. 3.

Table I.

\begin{tabular}{c|c|c|c|c|c|c|c}
\multicolumn{2}{c|}{} & \multicolumn{2}{c|}{ LLNL Stilbene } & \multicolumn{2}{c|}{ IO Stilbene } & \multicolumn{2}{c}{ EJ-309 Liquid } \\
\hline $\begin{array}{c}\text { Gamma Ray } \\
\text { Source }\end{array}$ & $\begin{array}{c}\text { Activity } \\
(\gamma / \mathrm{s})\end{array}$ & $\begin{array}{c}\mathrm{d}_{\gamma} \\
(\mathrm{cm})\end{array}$ & $\begin{array}{c}\mathrm{d}_{\mathrm{Cf}} \\
(\mathrm{cm})\end{array}$ & $\begin{array}{c}\mathrm{d}_{\gamma} \\
(\mathrm{cm})\end{array}$ & $\begin{array}{c}\mathrm{d}_{\mathrm{Cf}} \\
(\mathrm{cm})\end{array}$ & $\begin{array}{c}\mathrm{d}_{\gamma} \\
(\mathrm{cm})\end{array}$ & $\begin{array}{c}\mathrm{d}_{\mathrm{Cf}} \\
(\mathrm{cm})\end{array}$ \\
\hline${ }^{137} \mathrm{Cs}$ & $2.8 \times 10^{6}$ & 1.64 & 17.6 & 1.51 & 12.3 & 1.04 & 9.79 \\
${ }^{60} \mathrm{Co}$ & $5.0 \times 10^{6}$ & 2.14 & 15.4 & 2.53 & 13.8 & 1.94 & 11.4
\end{tabular}

\section{Data Analysis}

\subsection{Pulse Shape Discrimination by Charge Integration}

Charge integration was used for PSD with the stilbene and EJ-309 detectors. Figure 4 shows sample 400-mV pulses for each detector. Neutron pulses contain more light in their decaying (tail) region than gamma ray pulses; this behavior is quantified by integrating each pulse over both the full pulse length and the tail region after the peak. The integration ranges were optimized separately for each detector to maximize the PSD figure-of-merit [12]. The full pulse length ranged from 12 ns before the pulse amplitude until $300 \mathrm{~ns}$ after the pulse amplitude. The optimal tail start values (following the pulse maximum) were found to be $20 \mathrm{~ns}$ for the EJ-309 liquid, 24 ns for the LLNL stilbene, and $28 \mathrm{~ns}$ for the Inrad Optic stilbene. Pulses from the ${ }^{60} \mathrm{Co}^{252} \mathrm{Cf}$ and ${ }^{137} \mathrm{Cs}-{ }^{252} \mathrm{Cf}$ measurements were integrated in this manner for each detector, using a 42-keVee (440-keV proton) pulse amplitude threshold for the stilbene assemblies and a $60-\mathrm{keVee}(610-\mathrm{keV}$ proton) pulse amplitude threshold for the EJ-309 liquid.

(a)

(b)

Fig. 4.

\subsection{Neutron Detection Evaluation Criteria}

Neutron detection capabilities of each detector are evaluated by their intrinsic neutron efficiency Eff and gamma misclassification rate $M R$. These parameters are defined by Equations 1 and 2:

$$
\begin{gathered}
E f f=\frac{N_{C f}}{A_{C f} t \frac{\Omega}{4 \pi}} \\
M R=\frac{N_{\gamma}}{C_{\gamma}} .
\end{gathered}
$$


Here, $\mathrm{N}$ and $\mathrm{C}$ represent the respective neutron and total counts after background subtraction from the ${ }^{252} \mathrm{Cf}$ or gamma ray measurement (denoted by subscripts), $\mathrm{A}$ is the total neutron emission rate from the ${ }^{252} \mathrm{Cf}$ source, $\mathrm{t}$ is the measurement time, and $\Omega$ is the source-to-detector solid angle. Intrinsic efficiency was compared for each detector measuring ${ }^{252} \mathrm{Cf}$ with and without the highgamma field, and the misclassification rates were computed before and after each method of double-pulse cleaning. The energy threshold, PSD discrimination curve, and double-pulse cleaning parameters can be changed to favor one parameter at the expense of the other. For example, a higher energy threshold leads to fewer gammas misclassified as neutrons, but also lowered neutron efficiency.

\subsection{Approach to Double Pulse Cleaning}

Pulses from ${ }^{252} \mathrm{Cf}$ measurements with and without each gamma source are sorted by their tail and total integrals. These data are shown in Figure 5 for the Inrad Optic stilbene. The high-gamma field interferes with the charge integration technique, making it difficult to visually separate neutron pulses from gamma ray pulses. From Poisson statistics, the probability of two or more particles interacting in the same time window is given by

$$
\text { DoublePulseProbability }=1-e^{-\mu t} \text {, }
$$

where $\mu$ is the average count rate and $t$ is the time window length [13]. For a time window of 400 ns and average count rate of $100 \mathrm{kHz}$, the probability of multiple particles interacting in a single window is about 0.035 , yielding a double-pulse count rate of approximately $3500 \mathrm{~Hz}$. This doublepulse count rate is high when compared to a neutron count rate of approximately $100 \mathrm{~Hz}$. It should also be noted that these double pulses have a high tail integral relative to its total integral, making them "look like" neutrons, and thus must be removed from data analysis. The charge integration technique is most difficult for lower-energy pulses, and because ${ }^{137} \mathrm{Cs}$ has lower-energy gamma emissions than ${ }^{60} \mathrm{Co}$, the ${ }^{252} \mathrm{Cf}-{ }^{137} \mathrm{Cs}$ measurement was used to optimize double pulse cleaning. Settings for this measurement were then applied to the ${ }^{252} \mathrm{Cf}-{ }^{60} \mathrm{Co}$ and bare ${ }^{252} \mathrm{Cf}$ measurements.

(a) (b)

Fig. 5. (c)

\subsubsection{Fractional Double-Pulse Cleaning Technique}

The fractional double-pulse cleaning technique classified pulses as doubles if consecutive samples in the tail region increased by an amount that exceeded a set fraction of the pulse amplitude [14]. Figure 6a illustrates this technique on a clear double pulse. However, the technique also cleans out low-amplitude, good pulses. It is desired to maximize removal of double pulses and minimize removal of low-amplitude, good pulses. The fractional cleaning threshold was set to $6 \%$ of the pulse amplitude for each detector, which is a typical threshold that balances between thorough double-pulse cleaning and retaining of low-energy pulses. Increasing the fractional cleaning threshold to $7 \%$ or larger will increase the neutron efficiency, but will also clean fewer double pulses, misclassifying more gamma rays as neutrons. Figure $6 \mathrm{~b}$ shows the effect of implementing this fraction cleaning technique to the measured data of Figure 5c. Although there is 
greater clarity between the neutron and gamma regions, numerous double pulses still exist and cloud the space between the neutron and gamma ray regions at higher total integrals. The discrimination curve was chosen that separates between neutron and gamma rays for 1,200,000 pulses using the method described in section 3.3.3.

(a)

Fig. 6.

(b)

Table II shows the intrinsic efficiency and gamma misclassification rate for each detector before and after applying the fractional cleaning technique to each measurement. The fractional cleaning technique improves gamma misclassification rate by over 3 orders of magnitude in stilbene and over 2 orders of magnitude in EJ-309 liquid. However, its focus on removing lowenergy pulses leads to a loss of $20 \%$ efficiency when measuring ${ }^{252} \mathrm{Cf}$ only. Simply including the gamma source with ${ }^{252} \mathrm{Cf}$ leads to a reduction in intrinsic efficiency by approximately $13 \%$ because the high-gamma field forces cleaning of neutron pulses.

\begin{tabular}{c|c|c|c|c|c|c|c|c} 
& \multicolumn{9}{c}{ Eff } & \multicolumn{2}{c}{ Table II. } & \multicolumn{3}{c}{$\boldsymbol{M R}\left(\times 10^{-6}\right)$} \\
\cline { 2 - 9 } Detector & \multicolumn{2}{|c|}{${ }^{252} \mathrm{Cf}$} & $\begin{array}{c}{ }^{252} \mathrm{Cf}+ \\
{ }^{60} \mathrm{Co}\end{array}$ & $\begin{array}{c}{ }^{252} \mathrm{Cf}+ \\
{ }^{137} \mathrm{Cs}\end{array}$ & \multicolumn{2}{|c}{${ }^{60} \mathrm{Co}$} & ${ }^{137} \mathrm{Cs}$ \\
\cline { 2 - 9 } & $\begin{array}{c}\text { Before } \\
\text { Cleaning }\end{array}$ & $\begin{array}{c}\text { Fractional } \\
\text { Cleaning }\end{array}$ & $\begin{array}{c}\text { Fractional } \\
\text { Cleaning }\end{array}$ & $\begin{array}{c}\text { Before } \\
\text { Cleaning }\end{array}$ & $\begin{array}{c}\text { Fractional } \\
\text { Cleaning }\end{array}$ & $\begin{array}{c}\text { Before } \\
\text { Cleaning }\end{array}$ & $\begin{array}{c}\text { Fractional } \\
\text { Cleaning }\end{array}$ \\
\hline $\begin{array}{c}\text { LLNL } \\
\text { Stilbene }\end{array}$ & $18.9 \%$ & $14.9 \%$ & $12.7 \%$ & $13.7 \%$ & $12921(15)$ & $10.2(5)$ & $13641(15)$ & $6.0(4)$ \\
\hline $\begin{array}{c}\text { IO } \\
\text { Stilbene }\end{array}$ & $19.9 \%$ & $15.6 \%$ & $13.6 \%$ & $13.8 \%$ & $12840(15)$ & $6.6(5)$ & $14363(16)$ & $3.8(4)$ \\
\hline $\begin{array}{c}\text { EJ-309 } \\
\text { Liquid }\end{array}$ & $17.1 \%$ & $14.2 \%$ & $12.1 \%$ & $11.3 \%$ & $13375(15)$ & $46.2(1.0)$ & $14938(16)$ & $37.2(9)$
\end{tabular}

\subsubsection{Template Double-Pulse Cleaning Technique}

The template double-pulse cleaning technique made use of digitized pulse templates from data acquired from a low count rate experiment using ${ }^{252} \mathrm{Cf}$. The charge integration technique identified each ${ }^{252} \mathrm{Cf}$ pulse as a neutron or gamma pulse. These pulses were grouped by type and sorted by pulse amplitude, where pulses were averaged point-for-point to build gamma ray and neutron pulse templates for each detector as a function of pulse amplitude. The ${ }^{252} \mathrm{Cf}$ gamma ray and neutron pulse templates created for Inrad Optic stilbene are shown in Figures $7 \mathrm{a}$ and $7 \mathrm{~b}$.

The template technique compares pulses to their respective pulse template and cleans pulses that exceed the pulse template by more than a fixed threshold in their tail regions. Figure 7c shows examples of pulses removed by template cleaning. The low rise time of scintillation pulses, when sampled in 4-ns intervals, increases the likelihood that the true pulse amplitude is not sampled accurately. Because templates are created as a function of pulse amplitude, selecting the wrong template can remove good pulses. To minimize removal of good pulses and maximize removal of double pulses, the template threshold was chosen based on the standard deviation for every sample of the pulse templates, shown in Figure $7 \mathrm{~d}$ for Inrad Optic stilbene. The template threshold was 
chosen to be $15 \mathrm{mV}$ because the standard deviation for ${ }^{252} \mathrm{Cf}$ pulses stayed below $15 \mathrm{mV}$ (approximately yellow) throughout the template.

Figure 7e shows the effect of implementing this template cleaning technique to the measured data of Figure 5c, featuring a cleaner and more suppressed double pulse region. This technique also appears to preserve more low-energy neutron pulses than the fractional cleaning technique. However, there is now less separation between the neutron and gamma ray regions at lower energies. The discrimination line was chosen visually after implementing the hybrid cleaning technique, discussed in section 3.3.3, for 1,200,000 pulses.

(a)

(c) (b)

(d)

(e)

Fig. 7.

Table III shows the intrinsic efficiency and gamma misclassification rate after applying the template cleaning technique to each measurement for each detector. For stilbene, the template cleaning technique reduces the gamma misclassification rate by over 3 orders of magnitude in ${ }^{60} \mathrm{Co}$, similar to the fractional cleaning technique. Due to its focus on reducing higher-energy double pulses, only $3 \%$ of intrinsic efficiency is lost in stilbene when measuring bare ${ }^{252} \mathrm{Cf}$. However, the template cleaning technique is significantly worse with ${ }^{137} \mathrm{Cs}$ creating the high-gamma field than with ${ }^{60} \mathrm{Co}$. The higher light output threshold in the EJ-309 liquid allows for improvements in both efficiency and misclassification rate for EJ-309 liquid when using template cleaning over fractional cleaning. To consistently improve misclassification rate by over 3 orders of magnitude independent of gamma energy or detector type, a hybrid cleaning method is needed.

Table III.

\begin{tabular}{c|c|c|c|c|c}
\multirow{2}{*}{ Detector } & \multicolumn{4}{|c|}{ Eff } & \multicolumn{2}{c}{$\boldsymbol{M R}\left(\times 10^{-6}\right)$} \\
\cline { 2 - 6 } & ${ }^{252} \mathrm{Cf}$ & ${ }^{252} \mathrm{Cf}+{ }^{60} \mathrm{Co}$ & ${ }^{252} \mathrm{Cf}+{ }^{137} \mathrm{Cs}$ & ${ }^{60} \mathrm{Co}$ & ${ }^{137} \mathrm{Cs}$ \\
\hline $\begin{array}{c}\text { LLNL } \\
\text { Stilbene }\end{array}$ & $18.4 \%$ & $15.8 \%$ & $17.6 \%$ & $11.0(5)$ & $50.3(9)$ \\
\hline $\begin{array}{c}\text { IO } \\
\text { Stilbene }\end{array}$ & $19.2 \%$ & $16.8 \%$ & $17.5 \%$ & $10.4(1.0)$ & $60.1(4)$ \\
\hline $\begin{array}{c}\text { EJ-309 } \\
\text { Liquid }\end{array}$ & $15.3 \%$ & $12.9 \%$ & $12.2 \%$ & $13.1(5)$ & $28.0(7)$
\end{tabular}

\subsubsection{Hybrid Double-Pulse Cleaning Technique}

The proposed hybrid double-pulse cleaning technique uses the fractional cleaning technique to clean double pulses with amplitude of $180 \mathrm{keVee}$ or smaller, ensuring clear separation between the neutron and gamma ray regions, and the template cleaning technique to clean double pulses with amplitude greater than $180 \mathrm{keVee}$ to suppress the double pulse region. This will lower the expected neutron efficiency but will also improve the gamma misclassification rate relative to using either 
technique alone. Remaining pulses were compiled into PSD tail integral vs total integral distributions.

The discrimination line was obtained using a specialized MATLAB routine to bare ${ }^{252} \mathrm{Cf}$ data from each detector, inspired in part by [15]. This routine slices the distributions vertically, dividing pulses by total integral, using a slice width of $0.1 \mathrm{~V}$-ns. For the first 37 slices, pulses are histogrammed within each slice by their ratio of tail-to-total integrals to create separate gamma ray and neutron peaks such as that shown in Figure 8. Histograms are fit to a sum of two Gaussians, where each Gaussian represents particles classified as gamma rays or neutrons. A discrimination point is selected along the left edge of the second (neutron) peak when the edge equals a fraction of the fitted neutron peak maximum. This fraction was set more conservatively for lower-energy slices than higher-energy slices, since PSD is more difficult for lower-energy slices. The discrimination points from each slice were fit to a $2^{\text {nd }}$-order polynomial. The neutron peak fraction for each slice is given for each detector and slices used for generating the fit of each detector are given in Table IV.

Fig. 8 .

Table IV.

\begin{tabular}{c|c|c|c}
\multirow{2}{*}{ Slice Interval } & \multicolumn{3}{|c}{ Neutron Peak Fraction } \\
\cline { 2 - 4 } & LLNL Stilbene & IO Stilbene & EJ-309 Liquid \\
\hline 4 & 0.9 & 0.9 & -- \\
\hline $5-6$ & 0.6 & 0.6 & -- \\
\hline $7-8$ & 0.2 & 0.15 & 0.8 \\
\hline $9-10$ & 0.1 & 0.04 & 0.55 \\
\hline $11-13$ & 0.05 & 0.01 & 0.2 \\
\hline $14-16$ & 0.03 & 0.005 & 0.07 \\
\hline $17-18$ & 0.03 & 0.003 & 0.05 \\
\hline 19 & 0.02 & 0.002 & 0.04 \\
\hline $20-27$ & 0.02 & 0.002 & 0.02 \\
\hline $28-35$ & 0.03 & 0.002 & 0.02 \\
\hline $36-37$ & 0.03 & 0.002 & 0.01
\end{tabular}

This discrimination line and the fractional and template cleaning thresholds were applied to the bare ${ }^{252} \mathrm{Cf},{ }^{252} \mathrm{Cf}-{ }^{60} \mathrm{Co}$, and ${ }^{252} \mathrm{Cf}-{ }^{137} \mathrm{Cs}$ measurements. Figure 9 shows the tail integral vs. total integral surfaces obtained from 1,200,000 pulses from each detector in each measurement after hybrid cleaning. While some double pulses remain in each measurement, it is expected that most of these will be classified as gamma rays, and will not contribute to the calculated intrinsic neutron efficiency of each detector.
(a)
(b)
(c)
(d)
(e)
(g)
(h)
(i)
Fig. 9. 
Table V shows the intrinsic neutron efficiency and gamma misclassification rate after applying the hybrid cleaning technique to our experimental data. The hybrid cleaning technique consistently improves misclassification to the order of $10^{-6}$ in stilbene while losing approximately $20 \%$ neutron efficiency compared to template cleaning. This method is less effective in EJ-309 liquid, but still yields a $35 \%$ reduction in misclassification rate compared to fractional cleaning while sacrificing $16 \%$ efficiency compared to template cleaning. At a fixed count rate, misclassification was generally better when the detectors were exposed to ${ }^{60} \mathrm{Co}$ than ${ }^{137} \mathrm{Cs}$ because higher-energy gamma interactions are classified more accurately than lower-energy gamma interactions. In applications where the gamma emissions have energies lower than $662 \mathrm{keV}$, it is expected that the higher density of lower-energy gammas would lead to worse PSD performance and would require either more aggressive double-pulse cleaning parameters or lead shielding.

\begin{tabular}{c|c|c|c|c|c}
\multirow{2}{*}{ Detector } & \multicolumn{3}{|c|}{ Eff } & \multicolumn{2}{c}{$\boldsymbol{M R}\left(\times 10^{-6}\right)$} \\
\cline { 2 - 6 } & ${ }^{252} \mathrm{Cf}$ & ${ }^{252} \mathrm{Cf}+{ }^{60} \mathrm{Co}$ & ${ }^{252} \mathrm{Cf}+{ }^{137} \mathrm{Cs}$ & ${ }^{60} \mathrm{Co}$ & ${ }^{137} \mathrm{Cs}$ \\
\hline $\begin{array}{c}\text { LLNL } \\
\text { Stilbene }\end{array}$ & $14.4 \%$ & $12.2 \%$ & $13.2 \%$ & $3.2(3)$ & $4.1(3)$ \\
\hline $\begin{array}{c}\text { IO } \\
\text { Stilbene }\end{array}$ & $15.1 \%$ & $13.1 \%$ & $13.4 \%$ & $1.3(2)$ & $2.9(3)$ \\
\hline $\begin{array}{c}\text { EJ-309 } \\
\text { Liquid }\end{array}$ & $12.9 \%$ & $10.8 \%$ & $10.1 \%$ & $9.9(4)$ & $16.0(6)$
\end{tabular}

\section{Conclusions}

A new hybrid approach that uses fractional and template-based double-pulse cleaning was developed using digital data acquired using two stilbene detectors. Performance was compared to an EJ-309 liquid scintillation detector with the same scintillator dimensions and pulse readout system as the stilbene assemblies. All three detection systems were shown to be able to measure neutrons from an unshielded ${ }^{252} \mathrm{Cf}$ source in a field of at least 1000 incident gammas per incident ${ }^{252} \mathrm{Cf}$ neutron when using ${ }^{60} \mathrm{Co}$ or ${ }^{137} \mathrm{Cs}$ sources.

Despite using a lower light output threshold, the stilbene detectors and proposed data analysis technique led to gamma misclassification rates on the order of $10^{-6}$, a factor-of-5 better than the EJ309 liquid scintillation detector using the same technique. When measuring bare ${ }^{252} \mathrm{Cf}$, after hybrid double-pulse cleaning, the stilbene detectors exhibited intrinsic neutron efficiency of approximately $14.5 \%, 10 \%$ greater than the EJ-309 liquid detector. The efficiency decreases to approximately $13 \%$ when the gamma source is added since the presence of the source forces cleaning of additional neutron pulses overlapping with gamma pulses. We found a similar performance for neutron detection between the LLNL stilbene and Inrad Optic stilbene detectors (differences between their measured neutron efficiencies are on the order of 5\%, which is much smaller than the differences between stilbene and liquid scintillators). This was further verified in tests when PSD settings were set identically in each stilbene detector.

The reported intrinsic neutron efficiency and gamma misclassification rate are dependent on double-pulse cleaning and PSD settings. We found that lowering the fractional or template cleaning thresholds, increasing the energy threshold, or increasing neutron peak fractions for a more 
conservative discrimination line will improve the misclassification rate at the cost of intrinsic efficiency. On the other hand, using a template-based double-pulse cleaning approach is more desirable for applications where a misclassification of $10^{-5}$ is sufficient due to the improved intrinsic efficiency. Future work will seek to apply this hybrid double-pulse cleaning method to data acquired from plutonium samples with elevated gamma emission rates.

\section{Acknowledgment}

This work was funded in-part by the Consortium for Verification Technology under Department of Energy National Nuclear Security Administration award number DE-NA0002534.

\section{References}

1. D.A. Shea and D. Morgan, "The Helium-3 Shortage: Supply, Demand, and Options for Congress," Congressional research Service, 21 September 2010.

2. F.D. Brooks, Nuclear Instruments and Methods, 162 (1979), 477.

3. S. A. Pozzi, S. D. Clarke, M. Flaska, and P. Peerani, "Pulse Height Distributions of Neutron and Gamma Rays from Plutonium-Oxide Samples," Nuclear Instruments and Methods in Physics Section A, 608, 2, pp. 310-315, 2009.

4. H.W. Broek and C.E. Anderson, "The Stilbene Scintillation Crystal as a Spectrometer for Continuous FastNeutron Spectra," Review of Scientific Instruments, 31, 10, pp. 1063-1069, 1960.

5. L. Carman, N. Zaitseva, H. Paul Martinez, B. Rupert, I. Pawelczak, A. Glenn, H. Mulcahy, R. Leif, K. Lewis, and S. Payne, "The Effect of Material Purity on the Optical and Scintillation Properties of Solution-grown Transstilbene Crystals," Journal of Crystal Growth, 368, pp. 56-61, 2013.

6. N. Zaitseva, A. Glenn, L. Carman, H. P. Martinez, R. Hatarik, H. Klapper, and S. Payne, "Scintillation Properties of Solution-Grown Trans-Stilbene Single Crystals," Nuclear Instruments and Methods, in Physics Section A, 789, pp. 8-15, 2015.

7. Inrad Optics (2014), Stilbene Single Crystals, Retrieved January 15, 2015, from http://inradoptics.com/pdfs/datasheets/InradOptics_Datasheet_Stilbene_Final.pdf.

8. M. M. Bourne, S. D. Clarke, A. Enqvist, S. A. Pozzi, N. Zaitseva, and S. Payne, "Design of Handheld Stilbene System for Neutron Detection in a High-Gamma Field," Proceedings of INMM 55 ${ }^{\text {th }}$ Annual Meeting, Atlanta, GA, July 2014.

9. ProChem Inc, (2015), Retrieved July 6, 2015 from www.prochemonline.com.

10. ET Enterprises, (2011), Photomultipliers from ET Enterprises Ltd, Retrieved December 20, 2012 from http://www.et-enterprises.com/.

11. Eljen Technology (2014), EJ-309 Liquid Scintillator Pulse-Shape Discrimination Properties, Retrieved July 25, 2014 from http://www.eljentechnology.com/images/stories/Data_Sheets/Liquid_Scintillators/EJ309\%20data\%20 sheet.pdf.

12. I.A. Pawelczak, S.A. Ouedraogo, A.M. Glenn, R.E. Wurtz, and L.F. Nakae, "Studies of Neutron- $\gamma$ Pulse Shape Discrimination in EJ-309 Liquid Scintillator Using Charge Integration Method," Nuclear Instruments and Methods in Physics Section A, 711, pp. 21-26, 2013.

13. G.F. Knoll (2010), Radiation Detection and Measurement, Hoboken, NJ: John Wiley \& Sons, Inc., p. 810.

14. A.C. Kaplan, M. Flaska, A. Enqvist, J.L. Dolan, and S.A. Pozzi, "EJ-309 Pulse Shape Discrimination Performance with a High Gamma-ray-to-Neutron Ratio and Low Threshold," Nuclear Instruments and Methods in Physics Section A, 729, pp. 463-468, 2013.

15. J. K. Polack, M. Flaska, A. Enqvist, C. S. Sosa, C. C. Lawrence, and S. A. Pozzi, "An Algorithm for ChargeIntegration, Pulse-Shape Discrimination and Estimation of Neutron/Photon Misclassification in Organic Scintillators," Nuclear Instruments and Methods in Physics Section A (2015), http://dx.doi.org/10.1016/j.nima.2015.05.048.

Figure Captions 
Fig. 1. Stilbene scintillation cell and detector assembly.

Fig. 2. ${ }^{137}$ Cs calibration of the stilbene and the EJ-309 liquid scintillation detectors.

Fig. 3. Experimental setup showing ${ }^{252} \mathrm{Cf}$ source, gamma ray source, and stilbene detector assembly.

Table I. Measurement setups used for each detector. Distances are illustrated in Figure 3.

Fig. 4. Sample 250-mV pulses from stilbene (a) and EJ-309 liquid (b). Neutron and gamma rays pulses are identified using the charge integration technique.

Fig. 5. Tail integral vs. total integral for $1,200,000$ pulses of Inrad Optic stilbene from the ${ }^{252} \mathrm{Cf}(\mathrm{a}),{ }^{252} \mathrm{Cf}-{ }^{60} \mathrm{Co}(\mathrm{b})$, and ${ }^{252} \mathrm{Cf}-{ }^{137} \mathrm{Cs}$ (c) measurements.

Fig. 6. Fractional double pulse cleaning technique, illustrated for the Inrad Optic stilbene. This technique (a) removes double pulses while preserving as many low-amplitude good neutron pulses as possible. Setting the fractional threshold to $6 \%$ gives a new tail integral vs. total integral distribution for LLNL stilbene measuring ${ }^{252} \mathrm{Cf}+{ }^{137} \mathrm{Cs}(\mathrm{b})$.

Table II. Neutron intrinsic efficiency and gamma misclassification rate for each detector before and after fractional double-pulse cleaning. Stilbene data was processed at a 42-keVee threshold and EJ-309 liquid data was processed at a 60-keVee threshold. Statistical error is given in parentheses when not negligibly low.

Fig. 7. Double pulse cleaning using ${ }^{252} \mathrm{Cf}$ templates for Inrad Optic stilbene. Pulse templates for gamma rays (a) and neutrons (b) are determined from a low-count rate ${ }^{252} \mathrm{Cf}$ experiment. Pulses that exceed the template pulse by $15 \mathrm{mV}$ are removed (c). $15 \mathrm{mV}$ was chosen by examining the standard deviation of pulses in each template (d). This approach gives a new tail integral vs. total integral distribution for Inrad Optic stilbene measuring ${ }^{252} \mathrm{Cf}+{ }^{137} \mathrm{Cs}(\mathrm{e})$.

Table III. Neutron intrinsic efficiency and gamma misclassification rate for each detector after template double-pulse cleaning. Stilbene data was processed at a 42-keVee threshold and EJ-309 liquid data was processed at a 60-keVee threshold. Statistical error is given in parentheses when not negligibly low.

Fig. 8. Sample histogram of the ratio of tail-to-total integrals from a single slice obtained from ${ }^{252} \mathrm{Cf}$ pulses recorded by the Inrad Optic stilbene. Neutron pulses have a higher ratio than gamma ray pulses. The discrimination point is chosen along the left side of the neutron peak using a fraction of the peak maximum.

Table IV. Neutron peak fraction for each detector as a function of slice.

Fig. 9. PSD surfaces after hybrid double-pulse cleaning for $1,200,000$ pulses from ${ }^{252} \mathrm{Cf}$ bare,${ }^{252} \mathrm{Cf}+{ }^{60} \mathrm{Co}$, and ${ }^{252} \mathrm{Cf}+$ ${ }^{137}$ Cs respectively for LLNL stilbene (a-c), Inrad Optic stilbene (d-f), and EJ-309 liquid (g-i). Neutron and gamma regions can be visually distinguished and separated, although some double pulses still remain.

Table V. Neutron intrinsic efficiency and gamma misclassification rate for each detector after hybrid double-pulse cleaning. Stilbene data was processed at a 42-keVee threshold and EJ-309 liquid data was processed at a 60-keVee threshold. Statistical error is given in parentheses when not negligibly low. 


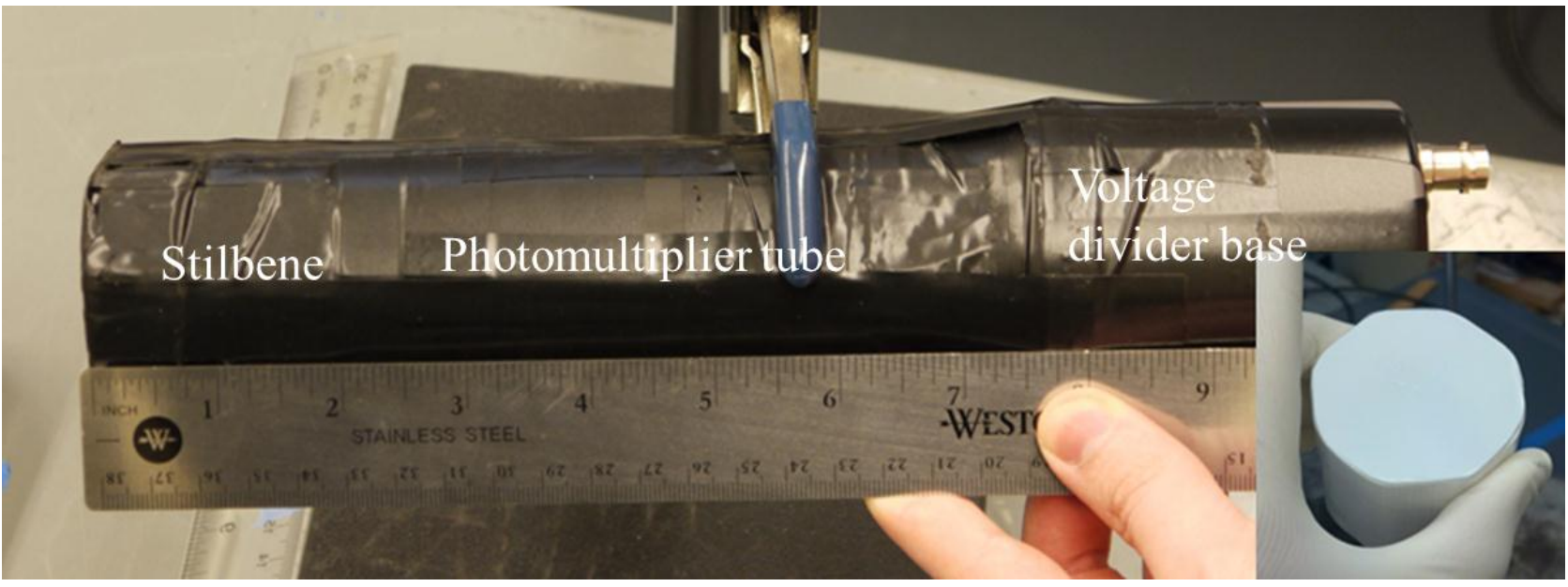




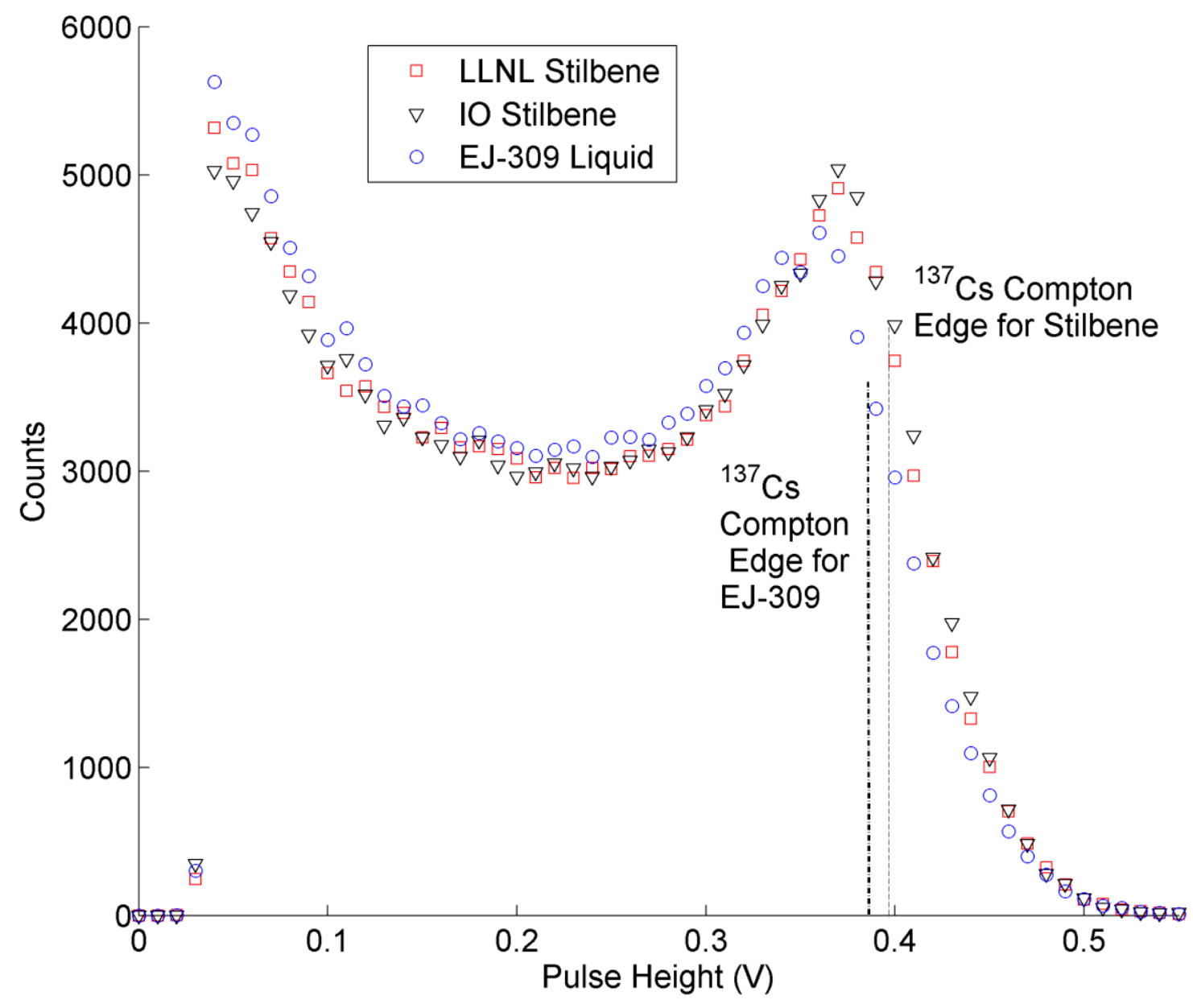




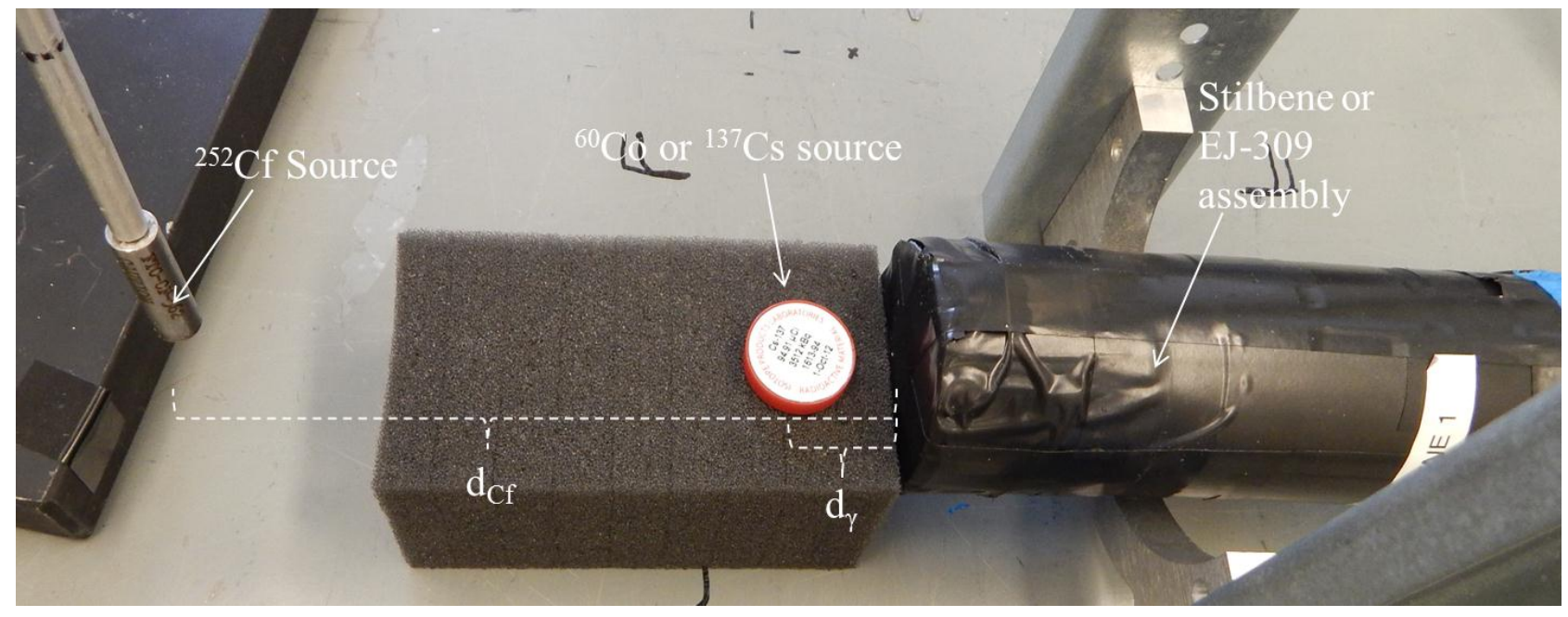




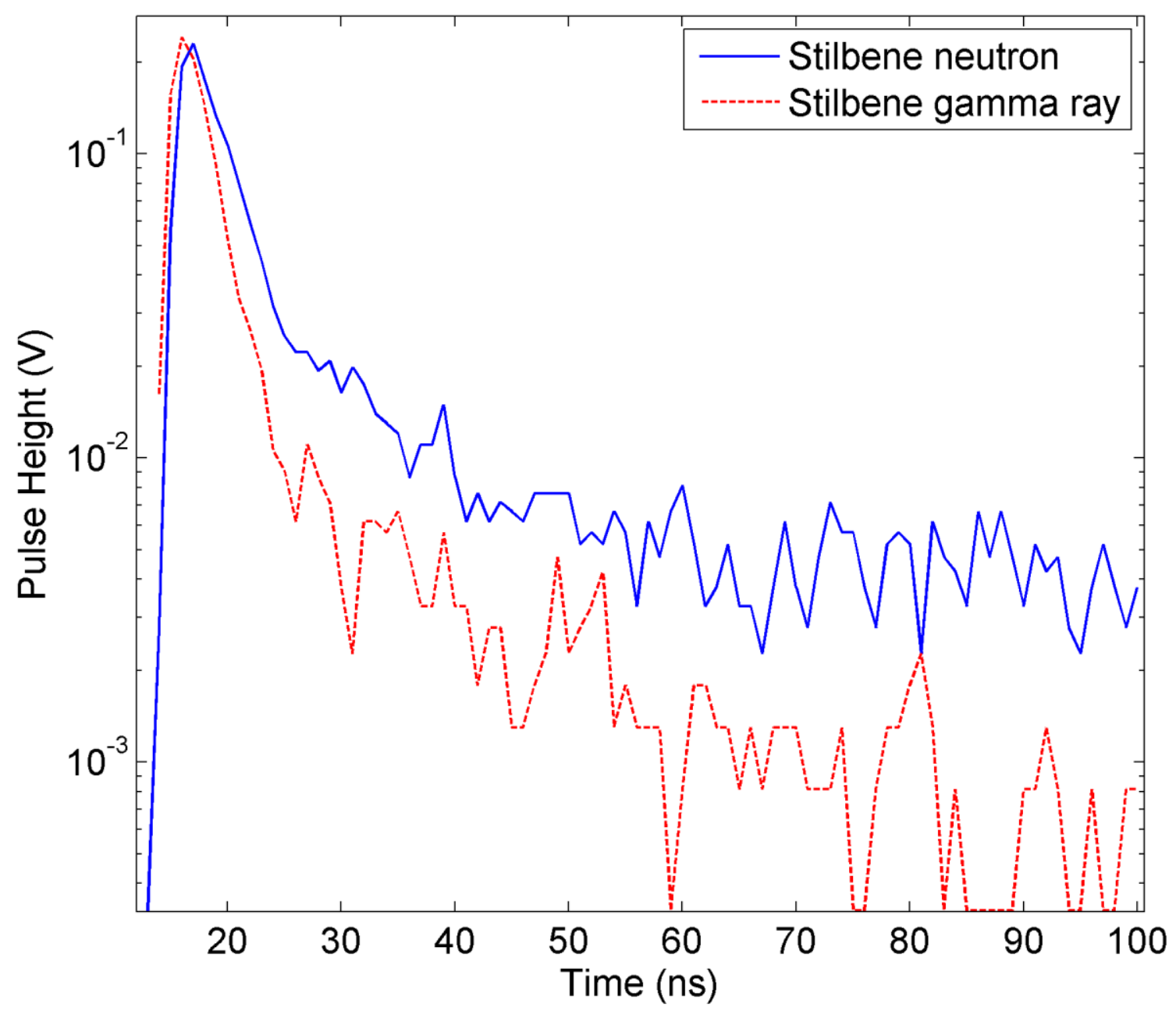




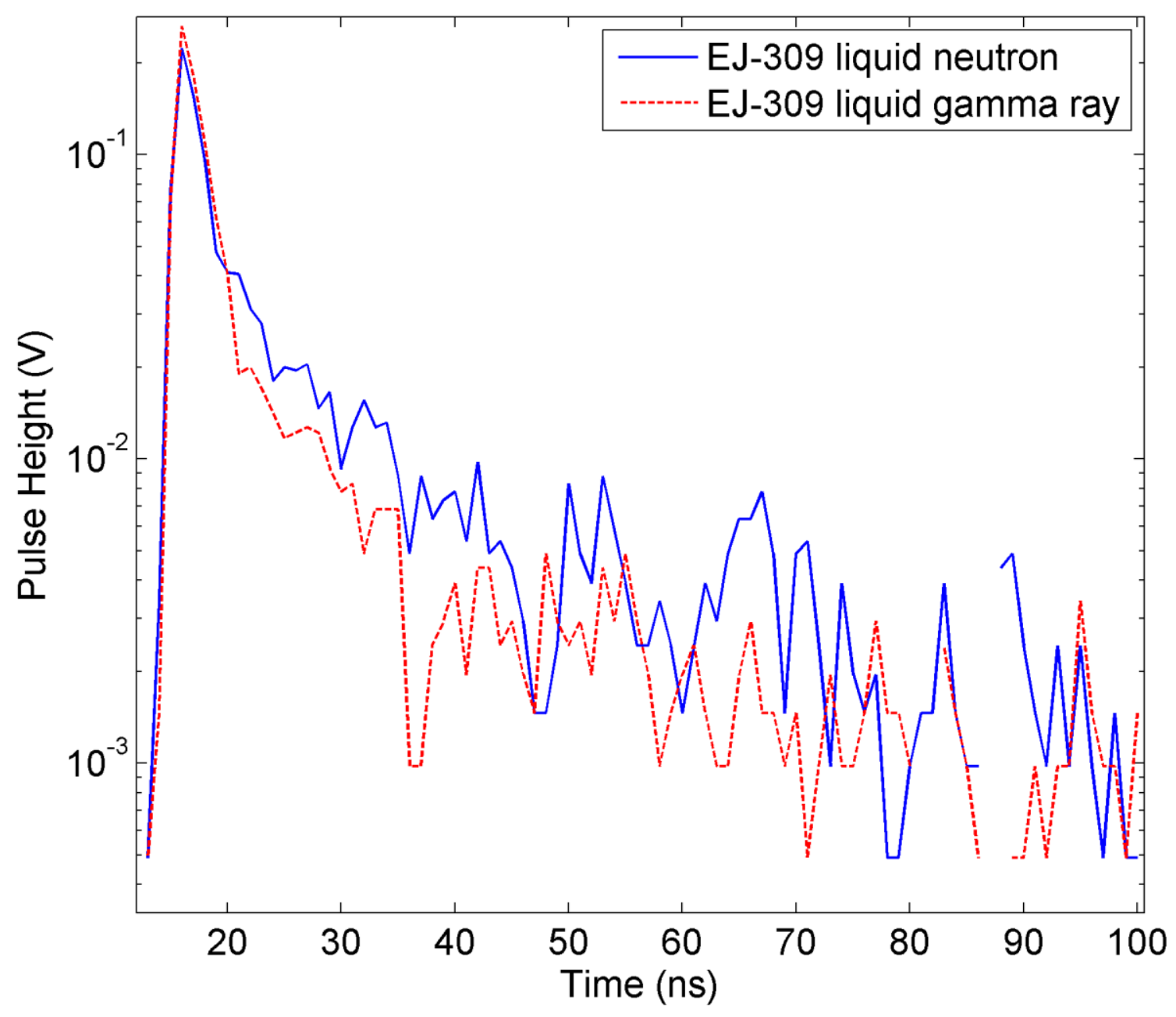




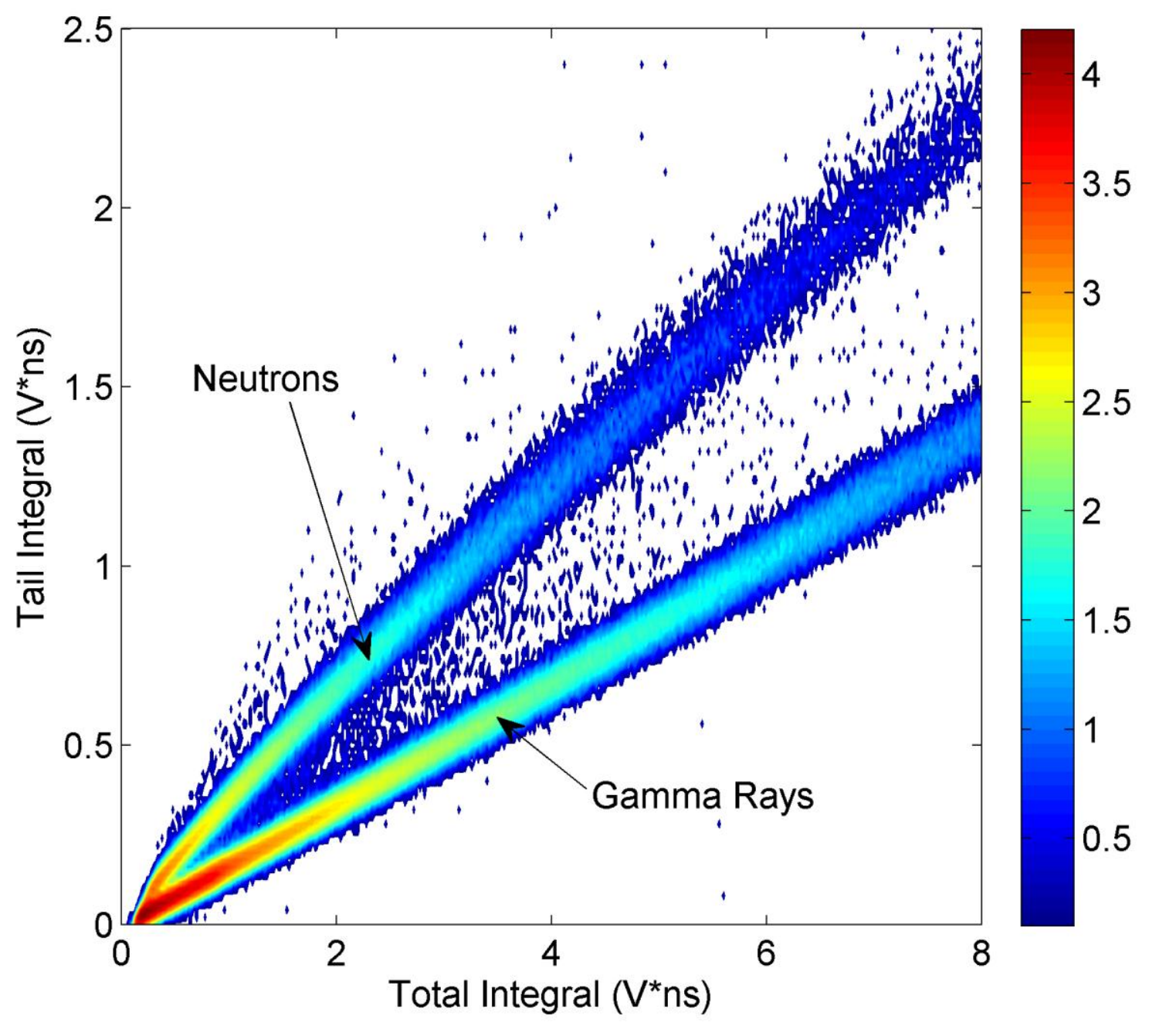




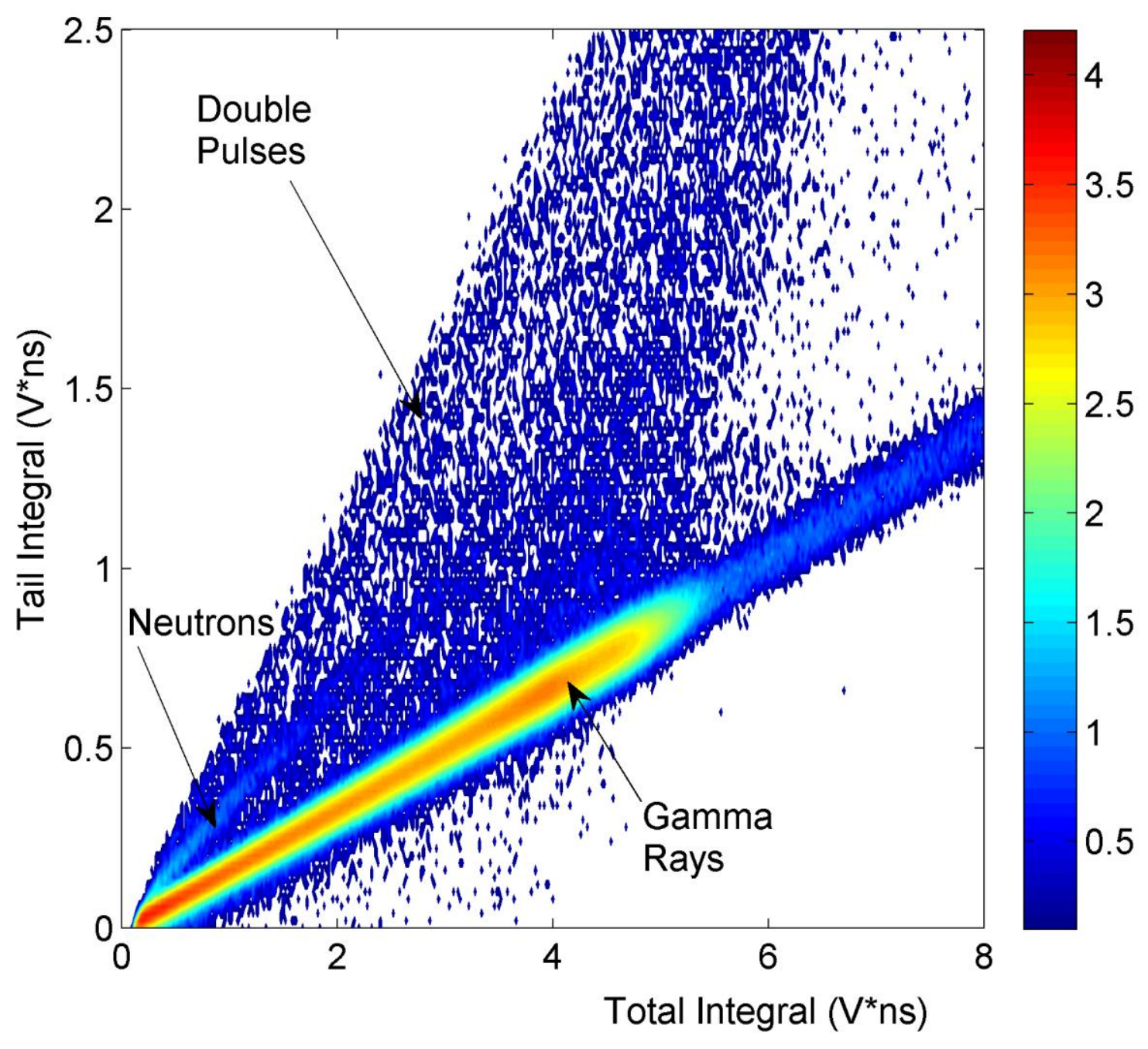




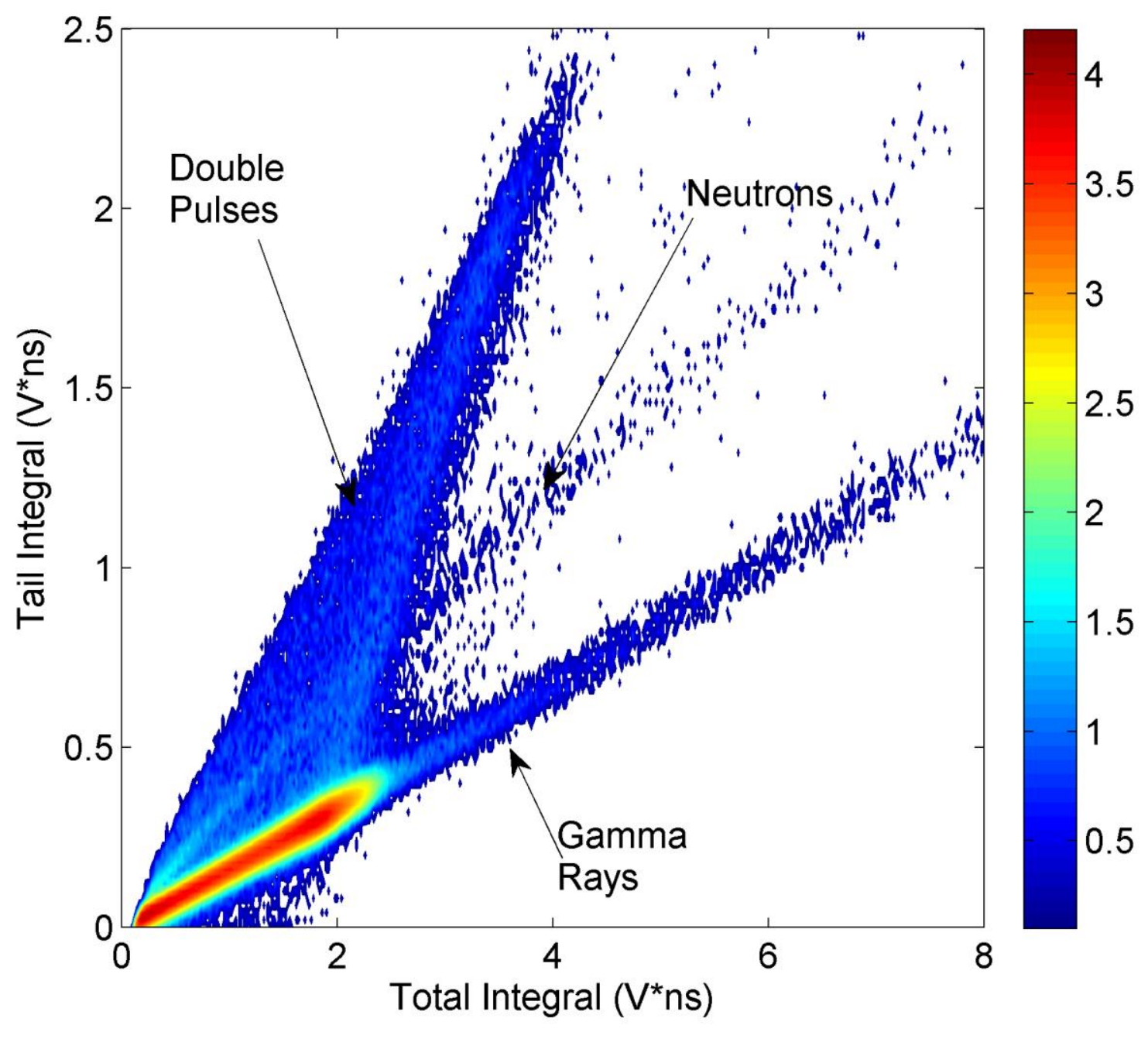




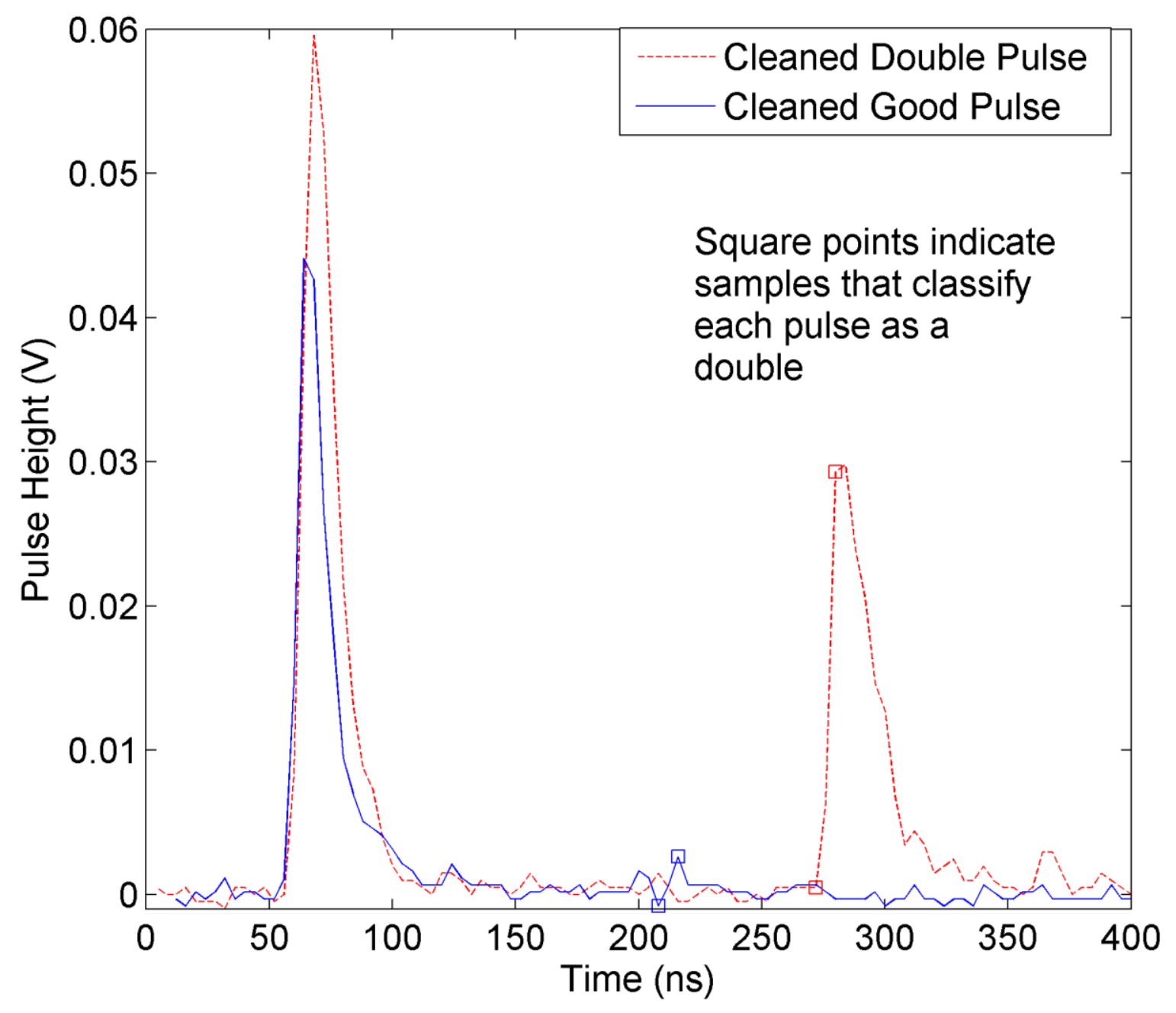




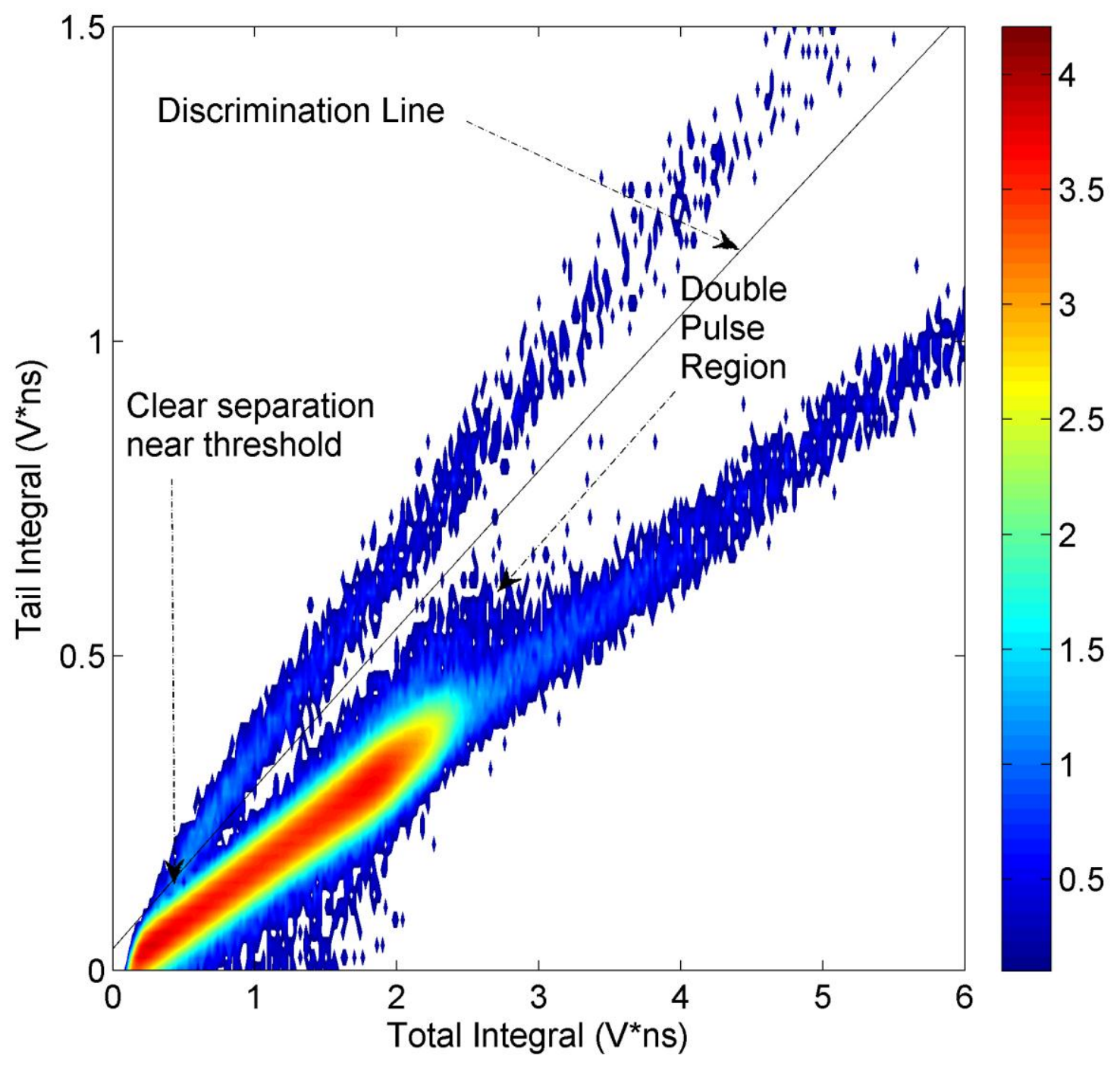




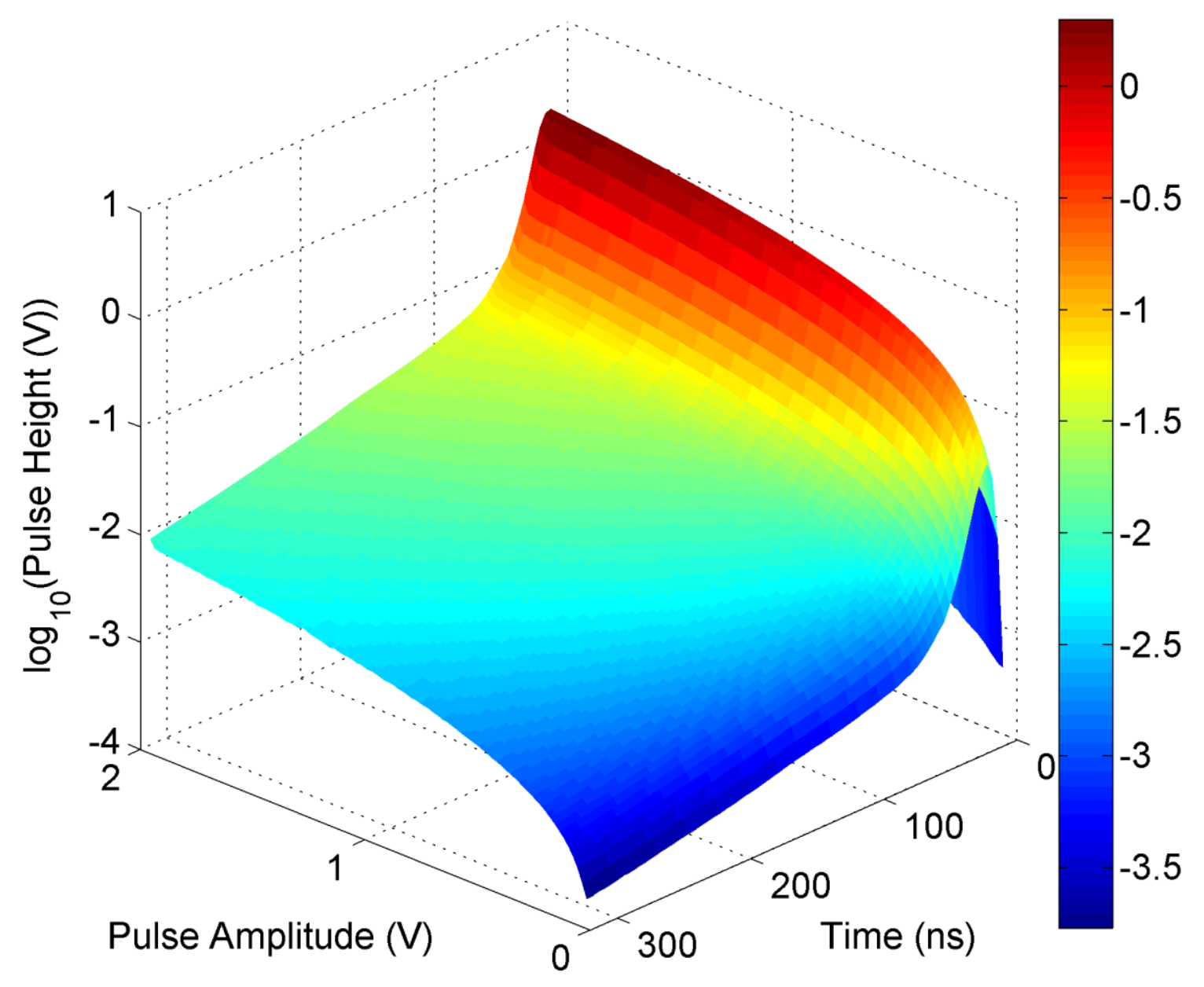




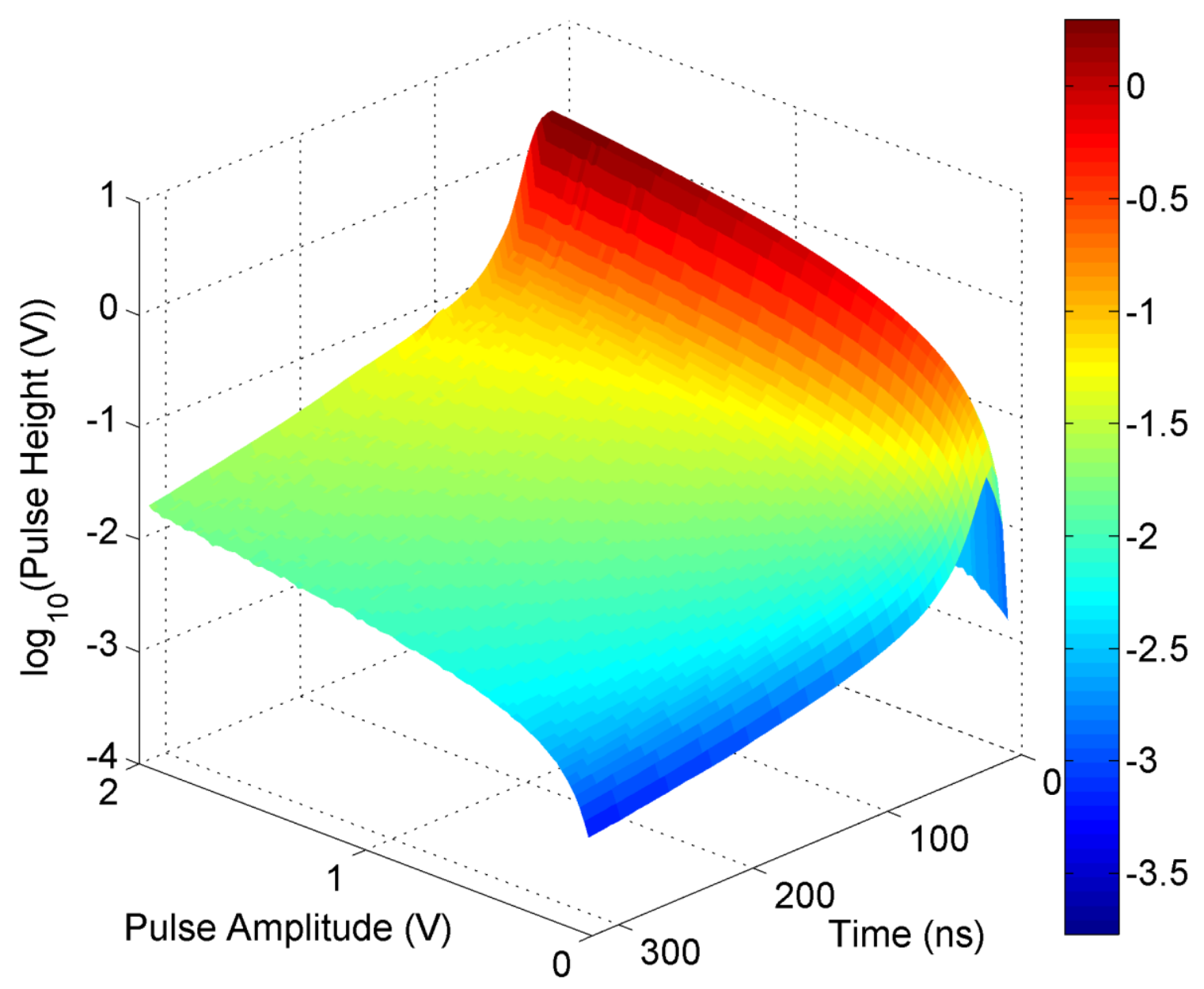




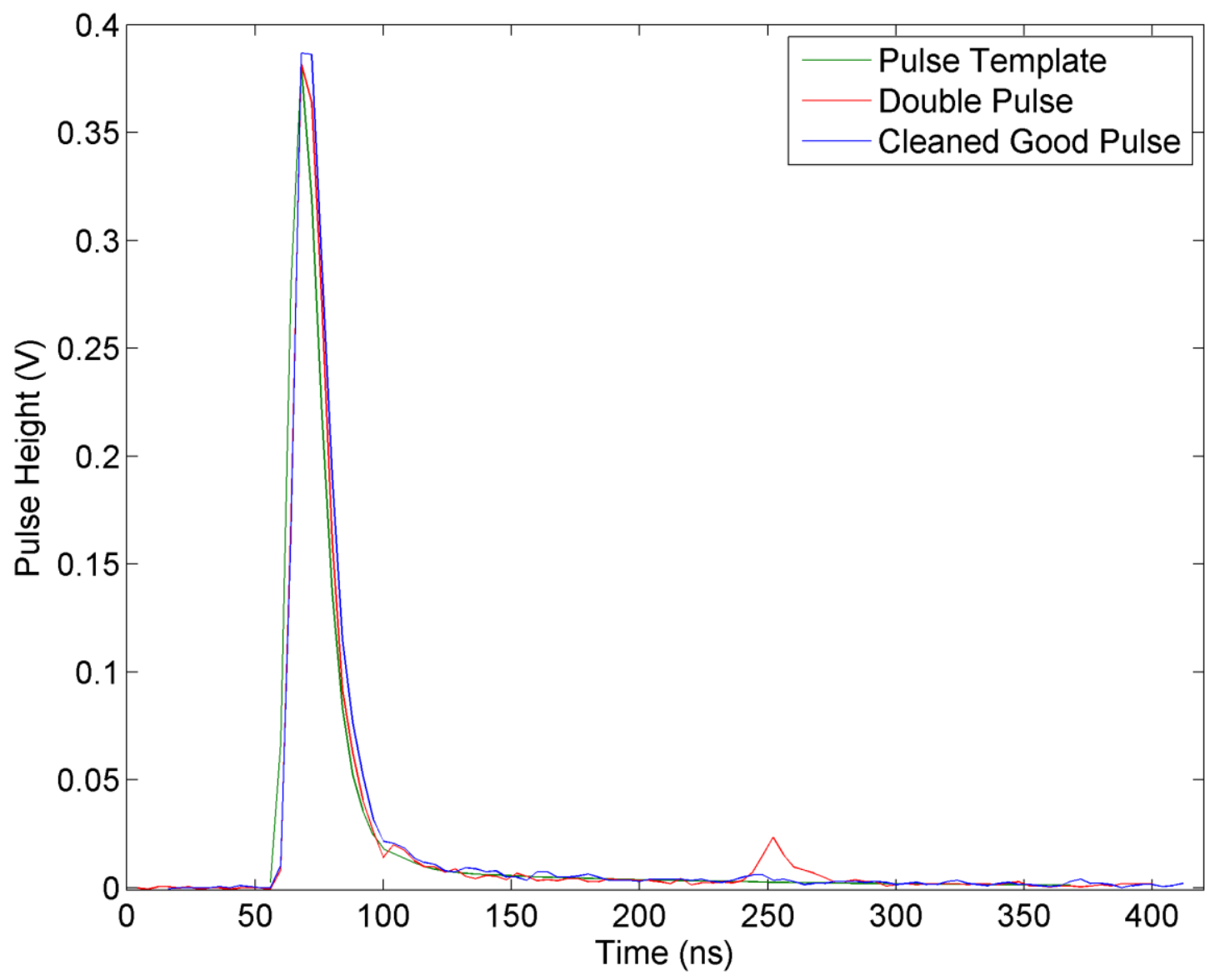




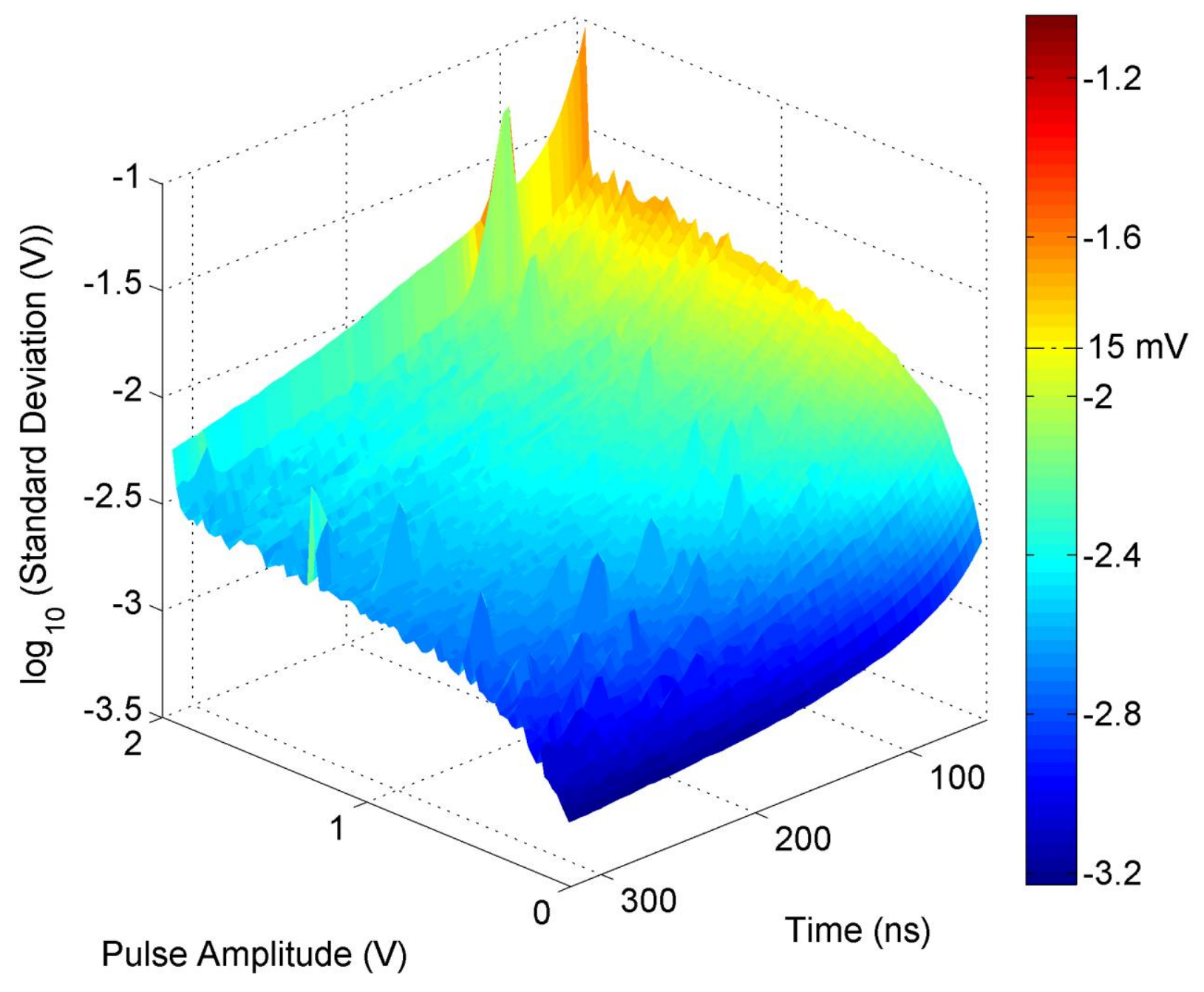




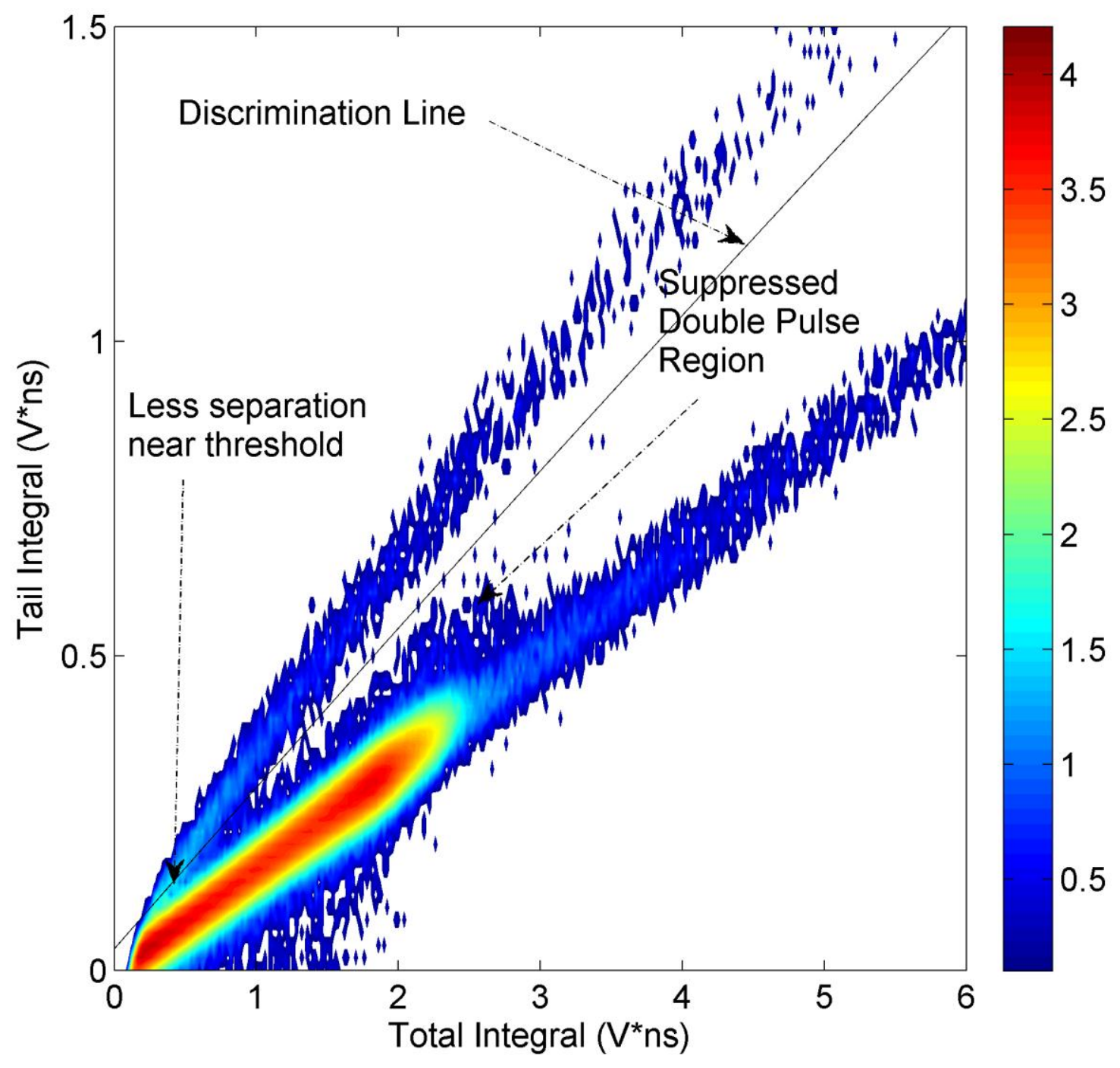




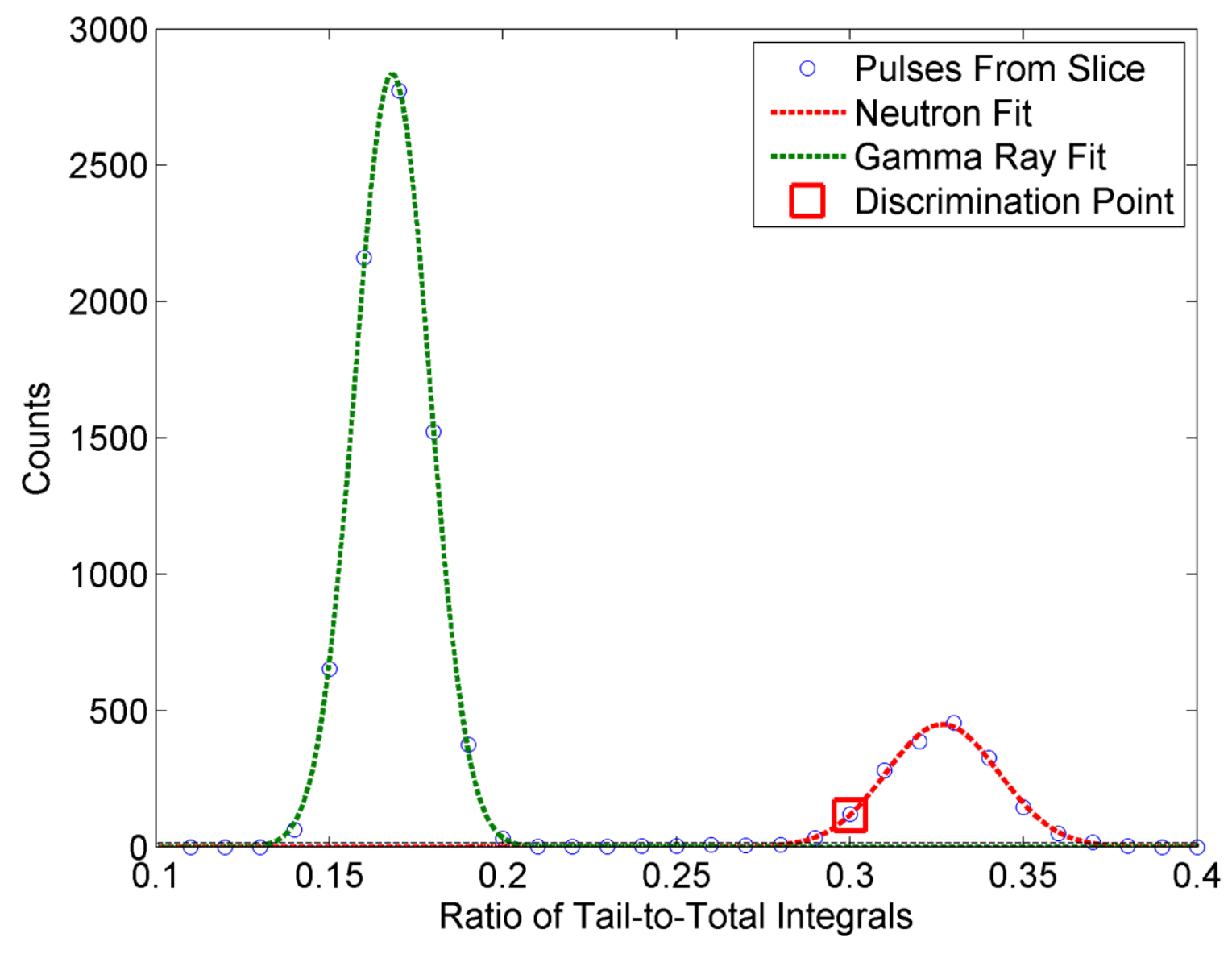




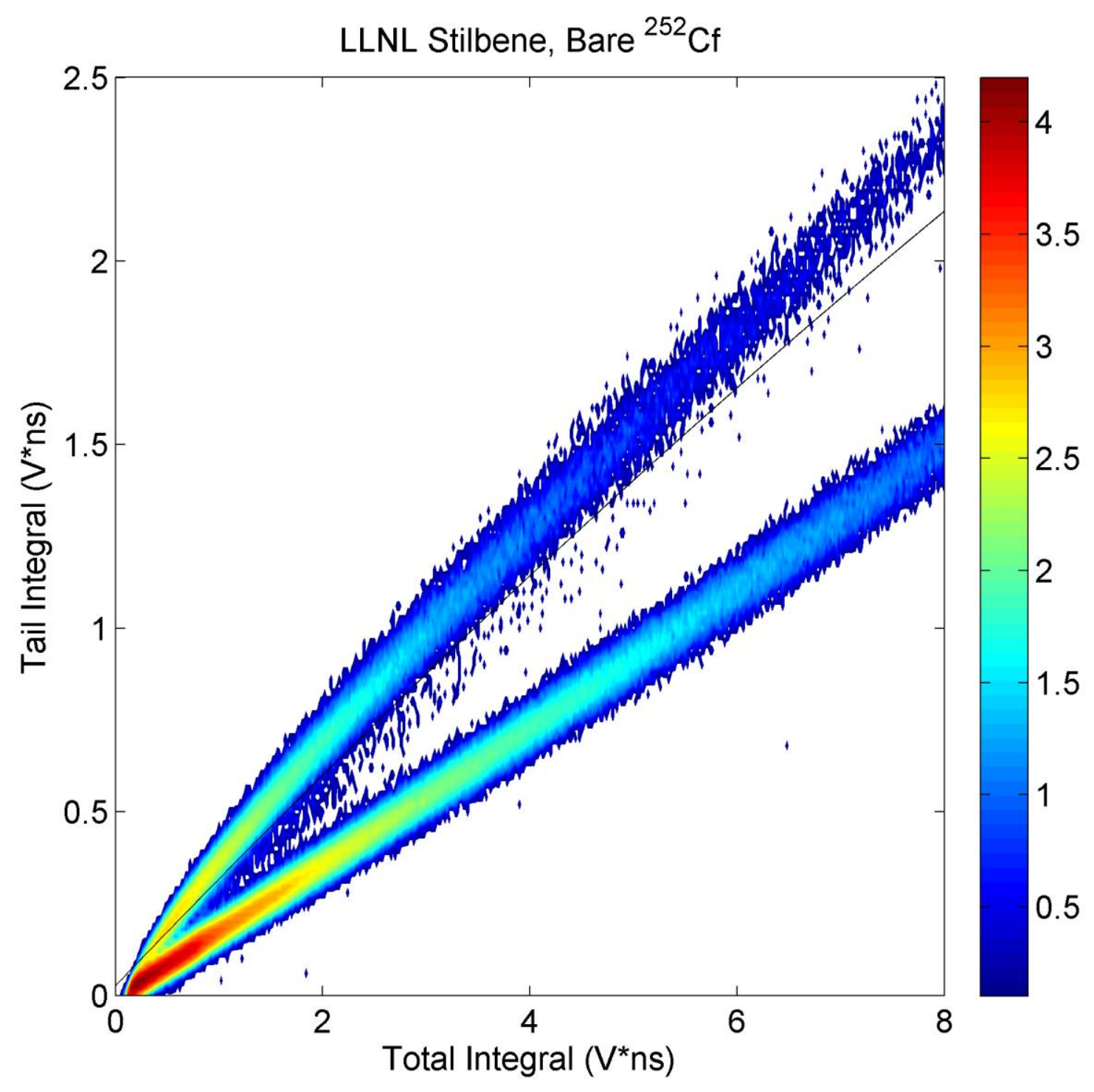




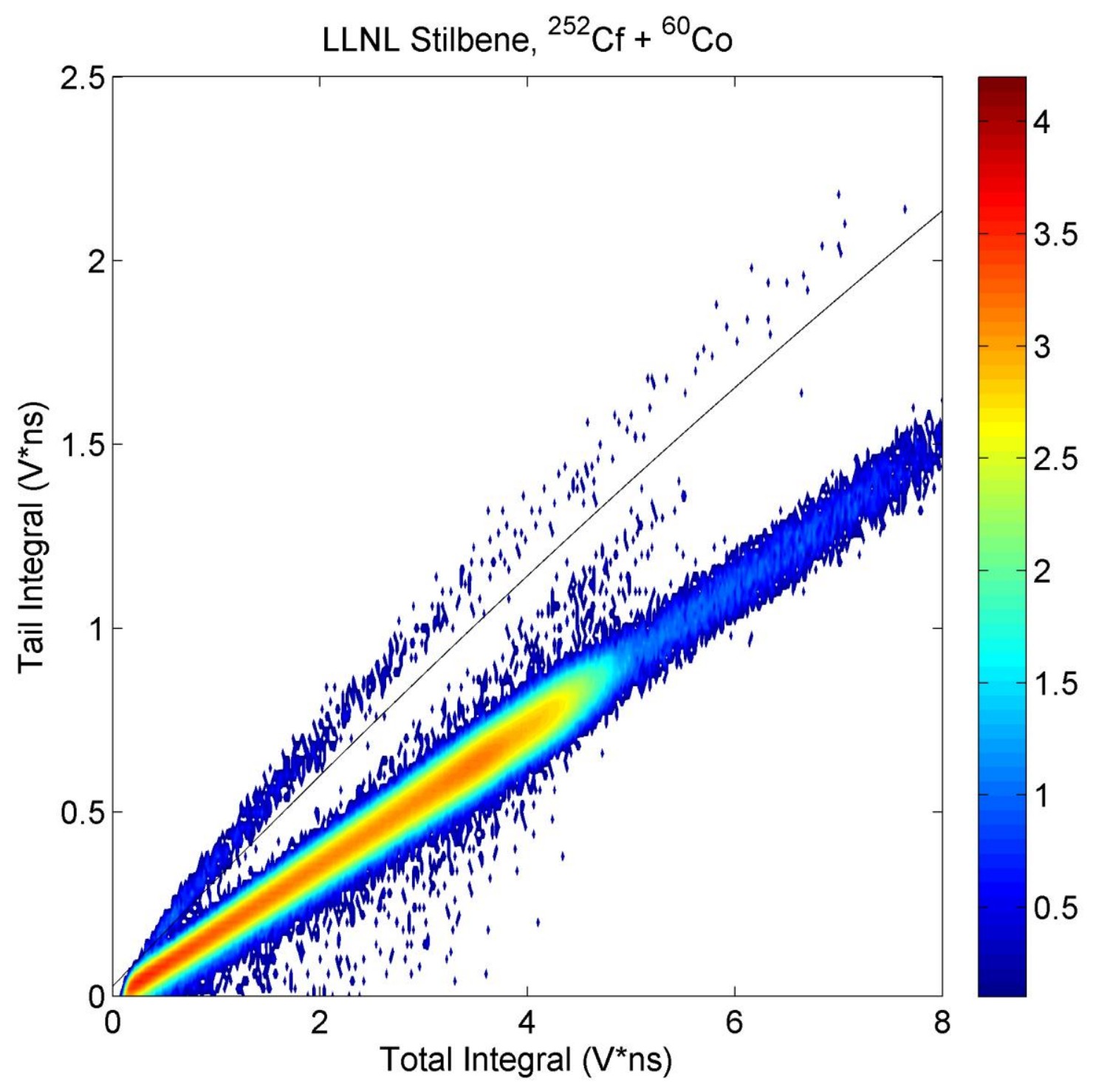




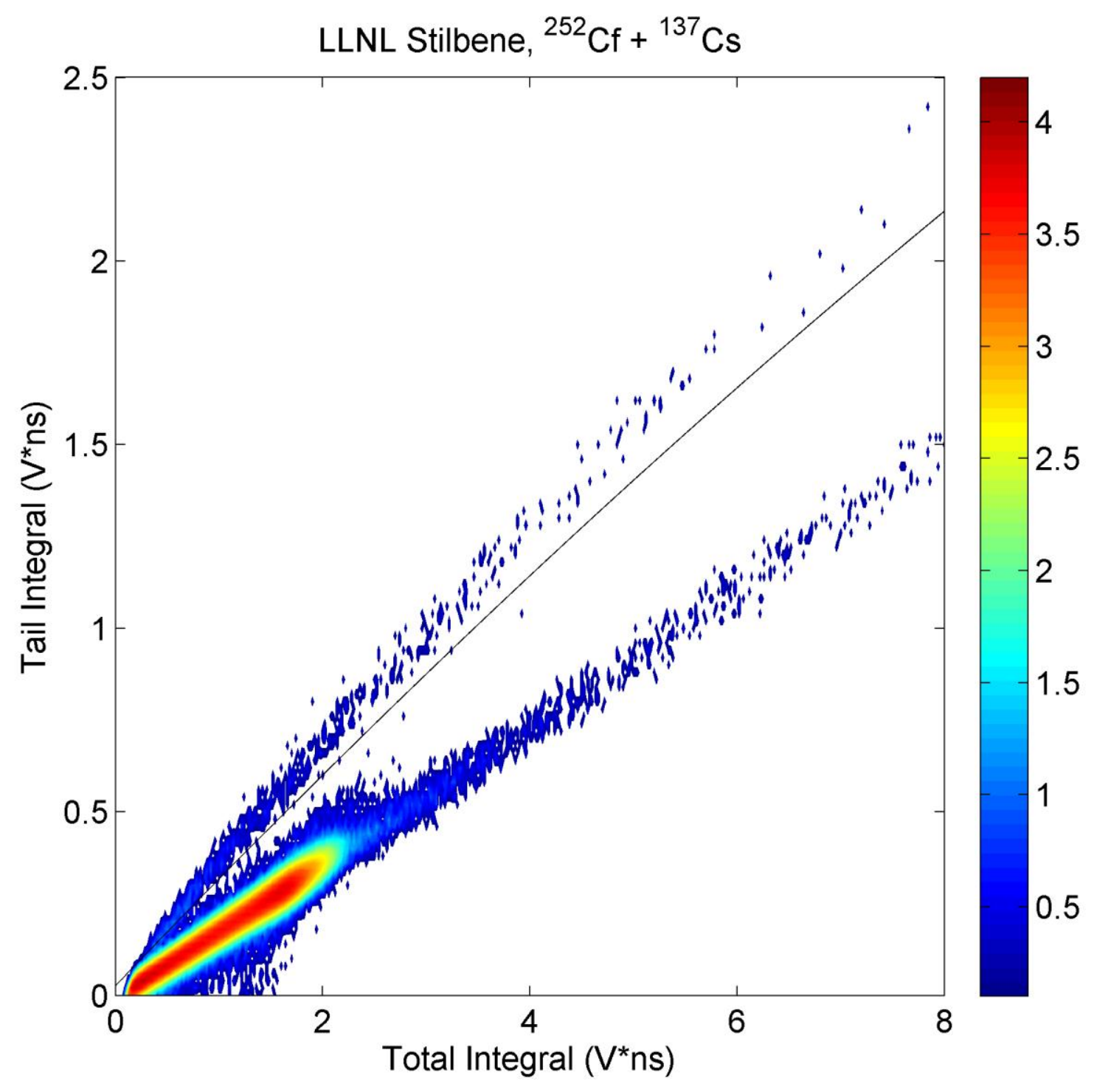




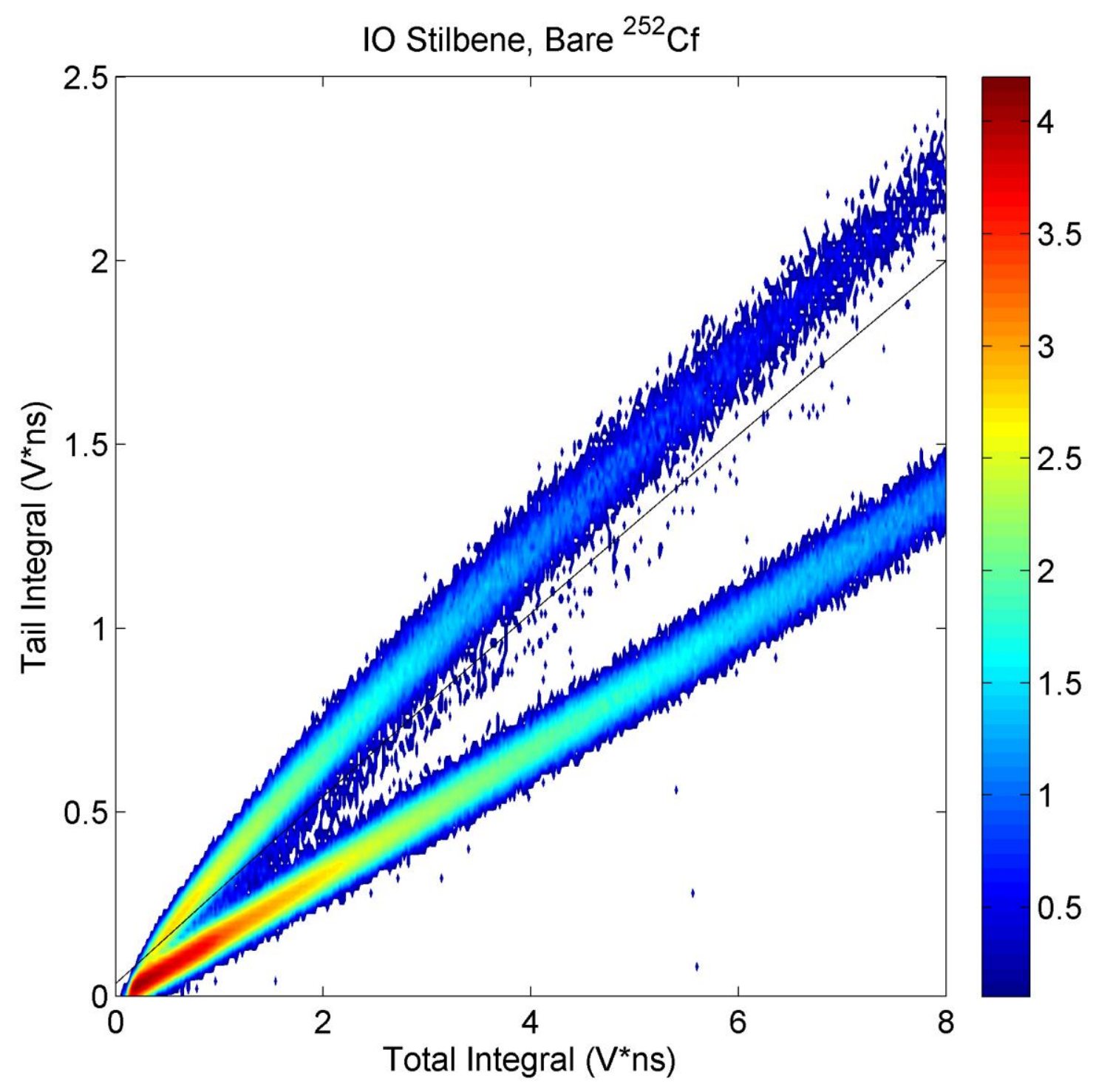




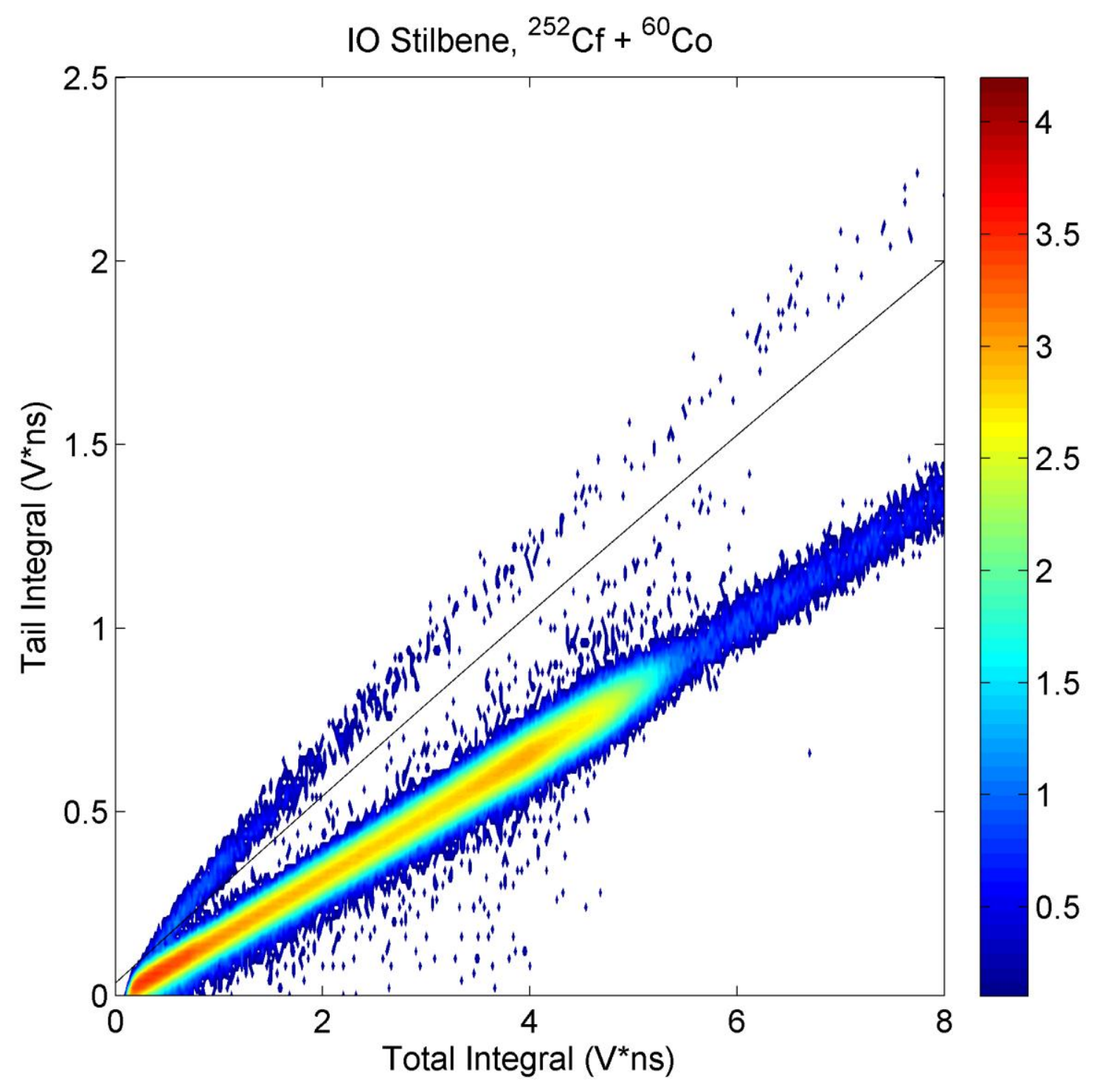




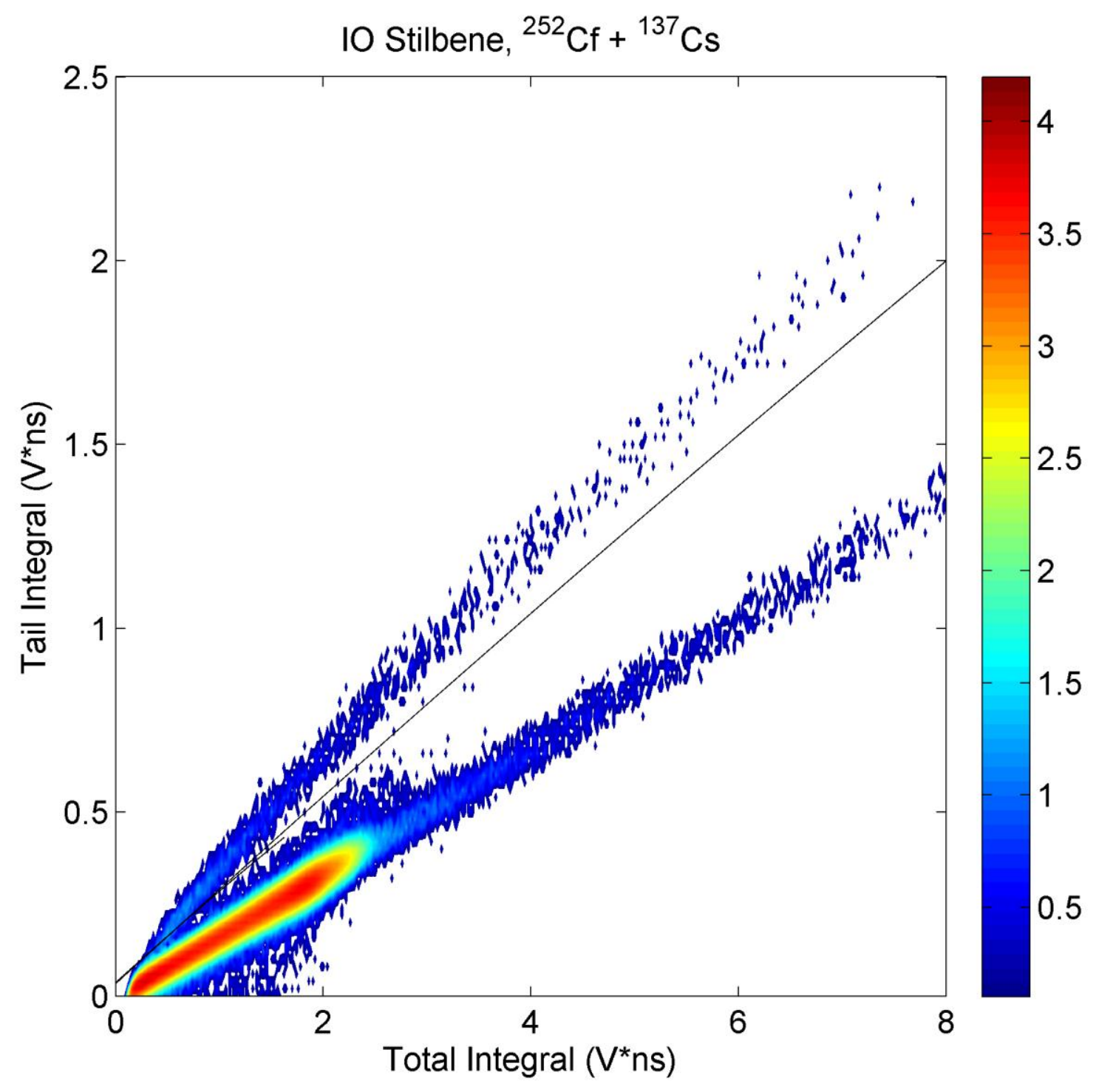




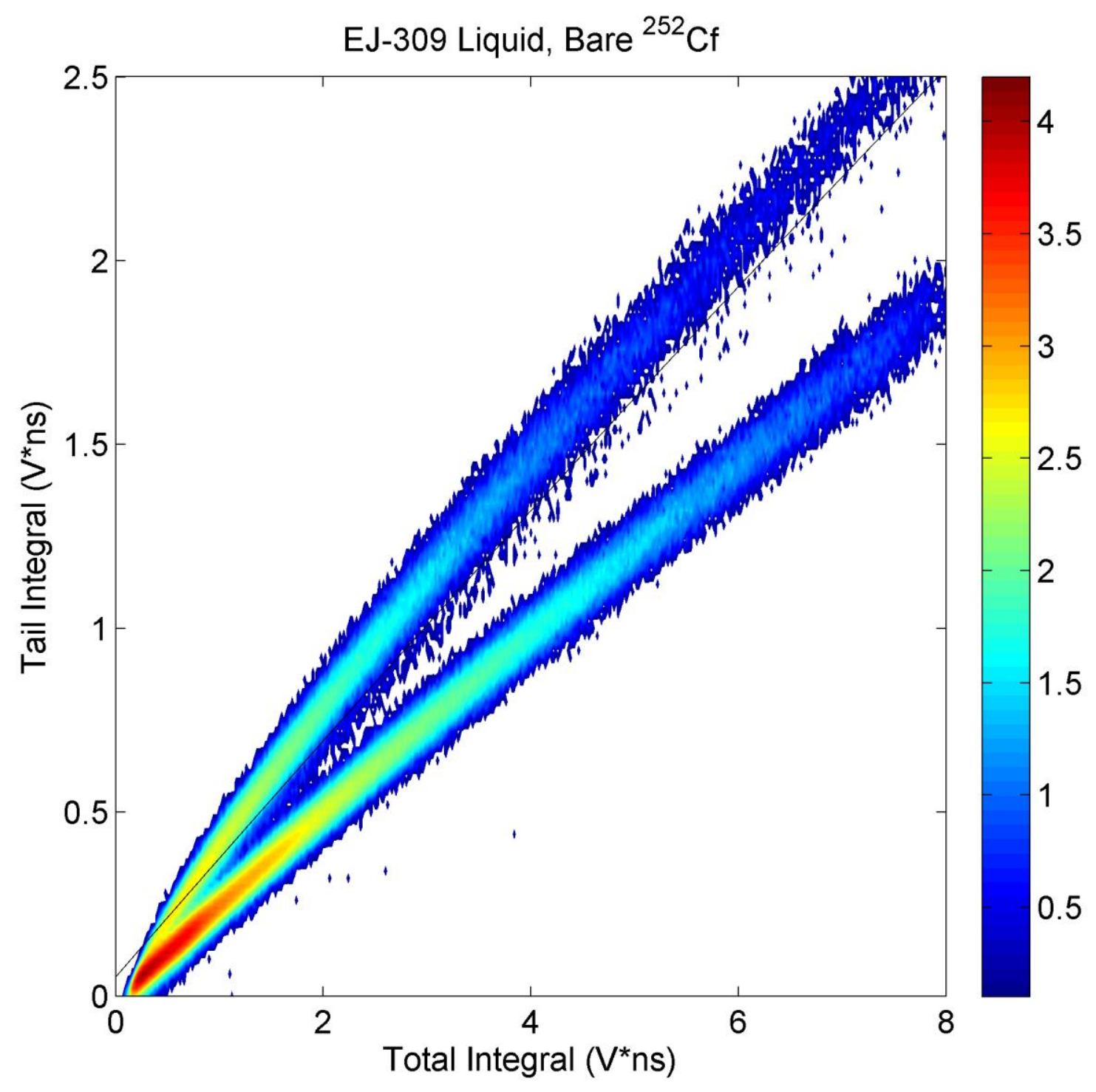




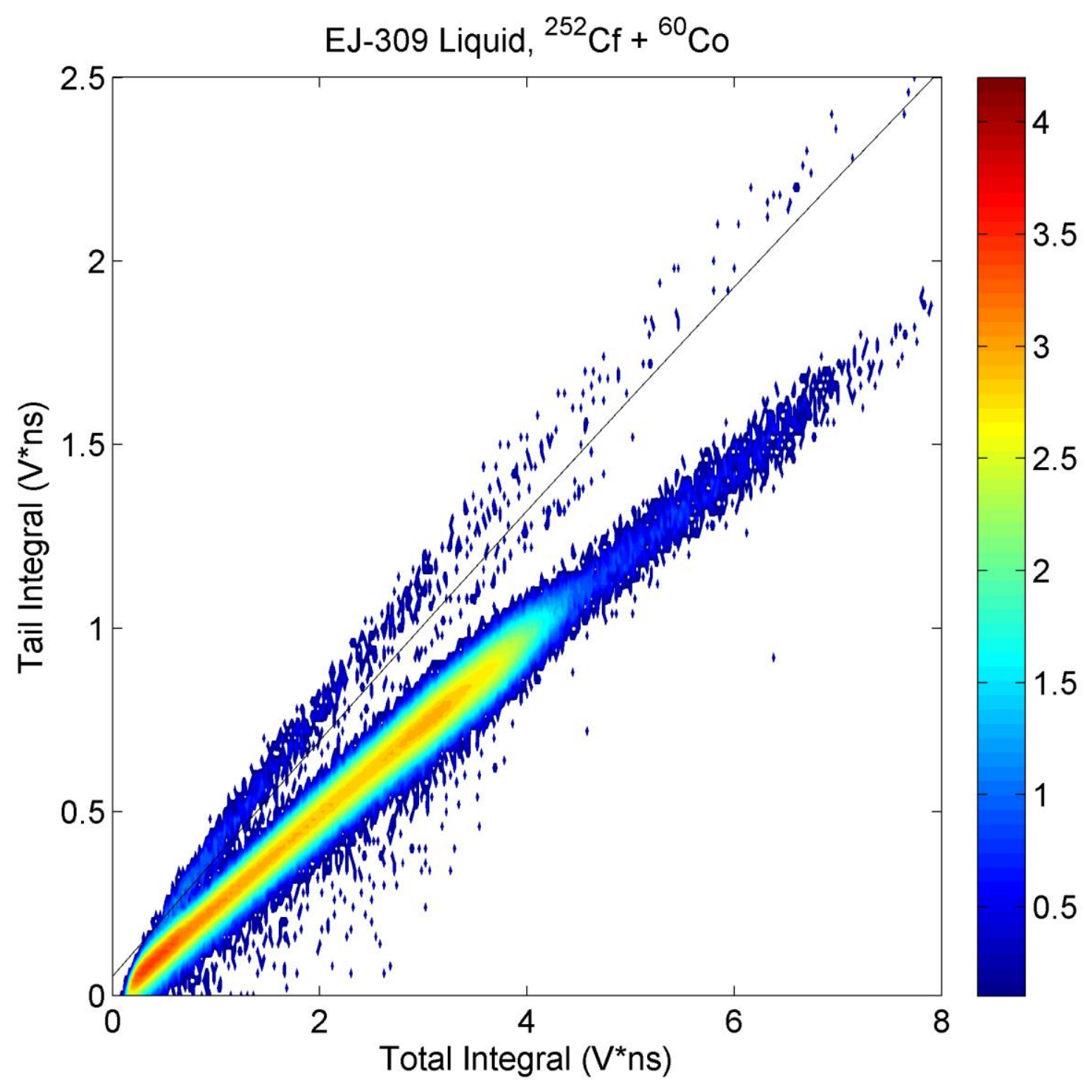




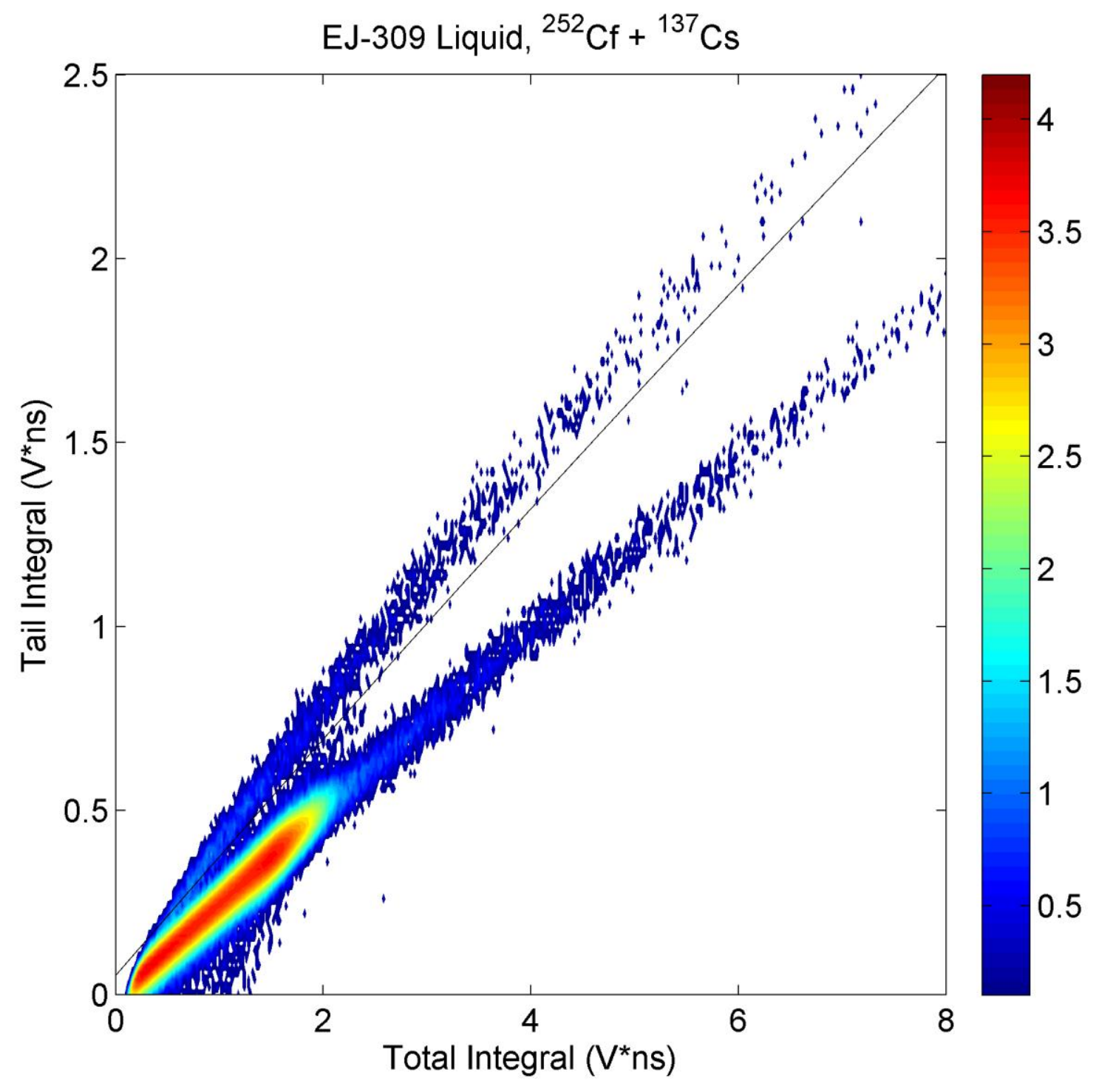

